\title{
UPSCALING OF A CLASS OF NONLINEAR PARABOLIC EQUATIONS FOR THE FLOW TRANSPORT IN HETEROGENEOUS POROUS MEDIA*
}

\author{
ZHIMING $\mathrm{CHEN}^{\dagger}$, WEIBING DENG ${ }^{\ddagger}$ AND HUANG YE§
}

Abstract. We develop an upscaling method for the nonlinear parabolic equation

$$
\partial_{t} b\left(u_{\varepsilon}\right)-\nabla \cdot\left(\mathbf{g}^{\varepsilon}\left(x, u_{\varepsilon}\right)+\mathbf{a}^{\varepsilon}\left(x, u_{\varepsilon}\right) \nabla u_{\varepsilon}\right)=f(x, t)
$$

which stems from the applications of the flow transport in porous media. Our direct motivation is the Richards equation which models the flow transport in unsaturated porous media. We provide a detailed convergence analysis of the method under the assumption that the oscillating coefficients are periodic. While such a simplifying assumption is not required by our method, it allows us to use homogenization theory to obtain the asymptotic structure of the solutions. Numerical experiments are carried out for the Richards equation of exponential model with periodic and randomly generated log-normal permeability to demonstrate the efficiency and accuracy of the proposed method.

Key words. Upscaling, nonlinear parabolic equation, heterogeneous porous media.

AMS subject classifications. $65 \mathrm{~F} 10,65 \mathrm{~F} 30$

\section{Introduction}

Over the past three decades a significant amount of research effort has been devoted to determining what are known as effective parameters in the modelling of subsurface flow and transport. The central difficulty is the accounting for the spatial variability in the parameters used to characterize the relevant physical properties of the natural porous media. In realistic situations, it is impossible to account explicitly for the spatial variability at all scales, due to computational resource limitations and/or the lack of data. Thus sophisticated geological and geostatistical modelling tools are used in practice to generate highly detailed medium parameters based on some site-specific measurements and experience from other sites. There exists a vast literature on the upscaling or homogenization techniques that lump the small-scale details of the medium into a few representative macroscopic parameters or effective parameters on a coarse scale which preserve the large-scale behavior of the medium and are more appropriate for reservoir simulations. We refer to the book of Christakos [6] for more information on the random field modeling of the natural porous medium parameters and the recent review papers $[27,32]$ on the existent upscaling techniques in the engineering literature. Jin.

*Received: May 29, 2005; accepted (in revised version): August 11, 2005. Communicated by Shi

${ }^{\dagger}$ LSEC, Institute of Computational Mathematics, Academy of Mathematics and Systems Science, Chinese Academy of Sciences, Beijing 100080, China (zmchen@lsec.cc.ac.cn).

This author was supported in part by China NSF under the grant 10025102 and by the National Basic Research Program of China under the grant 2005CB321701.

${ }^{\ddagger}$ Department of Mathematics, Nanjing University, Nanjing 210093, China and LSEC, Institute of Computational Mathematics, Academy of Mathematics and Systems Science, Chinese Academy of Sciences, Beijing 100080, China ( wbdeng@nju.edu.cn).

This author was supported in part by China Postdoctoral Science Foundation and the K. C. Wong Education Foundation, Hong Kong.

${ }^{\S}$ LSEC, Institute of Computational Mathematics, Academy of Mathematics and Systems Science, Chinese Academy of Sciences, and Graduate School of the Chinese Academy of Sciences, Beijing 100080, China (yeh@lsec.cc.ac.cn). 
The motivation of this paper is to solve the Richards equation (see, e.g., [1]), which models the flow transport in unsaturated porous media

$$
\frac{\partial \theta}{\partial t}-\frac{\partial K}{\partial x_{3}}-\nabla \cdot(K \nabla u)=f
$$

where $\theta$ is volumetric water content, $K$ is the absolute permeability tensor, $u$ is the fluid pressure, $x_{3}$ denotes the vertical coordinate in the medium, and $f$ stands for possible sources/sinks. The sources of nonlinearity of Richards equation come from the moisture retention function $\theta(u)$ and relative hydraulic conductivity function $K(\theta)$, respectively. Based on experimental results, many different functional relations have been proposed in the literature through various combinations of the dependent variables $\theta, u$ and $K$, and a certain number of fitting parameters (e.g., [3, 15, 17, $18,19])$. There are several widely known formulations of the constitutive relations such as the van Genuchten-Mualem model [18, 25], the Garder model [17], and the Haverkamp model [19]. For example, in the Garder model, also called the exponential model,

$$
\theta(u)=\theta_{r}+\left(\theta_{s}-\theta_{r}\right) e^{-\beta|u|}, \quad K(u)=K_{s} e^{-\alpha|u|},
$$

where $\theta_{r}$ and $\theta_{s}$ represent the residual water content and saturated water content respectively, $K_{s}$ is the saturated hydraulic conductivity, and $\alpha, \beta$ are parameters of the porous media. The Garder model has been widely used in the stochastic analysis of unsaturated flow in heterogeneous soils (see [24, 35]).

Recently a number of multiscale numerical methods, such as multiscale finite element method (MsFEM) [11], heterogeneous multiscale method (HMM) [10], and numerical homogenization method [13] have been proposed to solve the nonlinear problems. Originally, MsFEM is proposed for linear equations and its central idea is to incorporate the local small scale information of the leading order differential operator into the finite element bases. The common feature of the studies in [11] and [13] is that the computational complexity is proportional to the number of nodes of the fine-scale mesh which resolves the small scale spatial variability. However, the computational complexity can be significantly reduced if the homogenized fluxes are computed on the fly and these values can be stored and re-used if needed [11], [13]. Computation on the fly has advantages, especially if the fluxes depend on the gradients of the solution, because it only computes the selected values of the "homogenized fluxes".

The purpose of this paper is to develop a complete coarse grid algorithm for a class of nonlinear parabolic equations based on an upscaling procedure. The key idea is that we upscale the nonlinear constitutive relations such as the relationship between hydraulic conductivity versus pressure before we solve the nonlinear problems. We stress that the real significance of the method lies in its ability to solve the problems in coarse meshes. This is particularly advantageous when multiple simulations or realizations are necessary due to changes of boundary conditions or source functions for certain given fine micro-structures of the highly heterogeneous permeability of the porous media. Based on the homogenization theory, a sharp error estimate of the method can be established under the periodicity assumption. This assumption allows us to use the homogenization theory to obtain the asymptotic structure of the solutions. We emphasize that as pointed out in [9], the spatial periodicity assumption does not a priori restrict the applicability of the results only to media which do exhibit such strict repetitive spatial ordering in the properties of interest. The numerical experiments in $\S 5$ indicate that our method works fine for the well-accepted random log-normal permeability models in the engineering literature. 
Another new feature of our method is the way by which we deal with the nonlinear convection term. Our numerical procedure, as we show in the paper, shares a common element with the other multiscale methods, that is, the local information is coupled in the global formulation. The difference is the coupling method we use. Our local problem does not involve the convection term which is different from the MsFEM and the numerical homogenization approach introduced in [11, 13]. This idea has been introduced in a previous paper for the solute transport model in [4].

The outline of the paper is as follows. In Section 2 we introduce the upscaling method for the model problem. In Section 3 we review the homogenization results for the nonlinear parabolic problems with periodic coefficients. These results are the basis of our convergence analysis. Some error estimates for the homogenization equation are established in this section. In Section 4 we prove the error estimate for the upscaling method. In Section 5, we present some numerical examples for the Richards equation of the exponential model for both periodic and random log-normal coefficients to demonstrate the accuracy and efficiency of the method.

\section{Model problem and upscaling formulation}

Let $\Omega \subset \mathbb{R}^{d}, d=2,3$ be a bounded polyhedral domain with boundary $\partial \Omega$. We set $Q_{T}=\Omega \times(0, T), S_{T}=\partial \Omega \times(0, T)$ for $0<T<\infty$. Consider the following parabolic equation

$$
\begin{aligned}
\partial_{t} b\left(u_{\varepsilon}\right)-\nabla \cdot\left(\mathbf{g}^{\varepsilon}\left(x, u_{\varepsilon}\right)+\mathbf{a}^{\varepsilon}\left(x, u_{\varepsilon}\right) \nabla u_{\varepsilon}\right) & =f(x, t) & & \text { in } Q_{T}, \\
u_{\varepsilon}(x, t) & =0 & & \text { on } S_{T}, \\
u_{\varepsilon}(x, 0) & =u_{0}(x) & & \text { in } \Omega,
\end{aligned}
$$

where $\mathbf{a}^{\varepsilon}\left(x, u_{\varepsilon}\right)=\left(a_{i j}^{\varepsilon}\left(x, u_{\varepsilon}\right)\right)$ is a symmetric, positive definite, bounded tensor:

$$
\lambda|\xi|^{2} \leq a_{i j}^{\varepsilon}(x, s) \xi_{i} \xi_{j} \leq \Lambda|\xi|^{2} \quad \forall \xi \in \mathbb{R}^{d}, x \in \bar{\Omega}, s \in \mathbb{R}
$$

for some positive constants $\lambda$ and $\Lambda$, and $\mathbf{g}^{\varepsilon}\left(x, u_{\varepsilon}\right)=\left(g_{i}^{\varepsilon}\left(x, u_{\varepsilon}\right)\right)$ is a bounded vector. $\varepsilon$ is the characteristic length representing the small scale variability of the media. We also assume that $\frac{\partial}{\partial s} a_{i j}^{\varepsilon}(x, s), \frac{\partial}{\partial s} g_{i}^{\varepsilon}(x, s)$ are uniformly bounded and $b(s)$ satisfies

$$
0<b_{1} \leq b^{\prime}(s) \leq b_{2}<\infty, \quad b^{\prime \prime}(s)<C \quad \forall s \in \mathbb{R} .
$$

Define the space

$$
\mathcal{W}=\left\{u: u \in L^{2}\left(0, T ; H_{0}^{1}(\Omega)\right), u \in H^{1}\left(0, T ; H^{-1}(\Omega)\right)\right\} .
$$

The variational problem of $(2.1)$ is to seek $u_{\varepsilon}(x, t) \in \mathcal{W}$, for almost every $t \in(0, T)$, $u_{\varepsilon}(\cdot, t) \in H_{0}^{1}(\Omega)$ such that $u_{\varepsilon}(x, 0)=u_{0}(x)$ in $\Omega$, and

$$
\left(\partial_{t} b\left(u_{\varepsilon}\right), w\right)+\left(\mathbf{g}^{\varepsilon}\left(x, u_{\varepsilon}\right)+\mathbf{a}^{\varepsilon}\left(x, u_{\varepsilon}\right) \nabla u_{\varepsilon}, \nabla w\right)=(f, w) \quad \forall w \in H_{0}^{1}(\Omega) .
$$

Here and henceforth, $(\cdot, \cdot)$ stands for the inner product of $L^{2}(\Omega)$ or the duality pairing between $H^{-1}(\Omega)$ and $H_{0}^{1}(\Omega)$.

Instead of solving (2.4) on a fine mesh with a mesh size resolving the small scale variability $\varepsilon$, the basic idea of the upscaling methods is to solve the homogenized or upscaled equation

$$
\left(\partial_{t} b(u), w\right)+\left(\mathbf{g}^{*}(x, u)+\mathbf{a}^{*}(x, u) \nabla u, \nabla w\right)=(f, w) \quad \forall w \in H_{0}^{1}(\Omega) .
$$


The homogenized coefficients $\mathbf{a}^{*}(x, s), \mathbf{g}^{*}(x, s)$, for $s \in \mathbb{R}$, can be computed analytically from $\mathbf{a}^{\varepsilon}, \mathbf{g}^{\varepsilon}$ if they are periodic with respect to the second variable (see Section 3 below). Unfortunately, for practical natural porous media, such analytical formulae do not exist. In the following we shall develop a way to compute the nonlinear relations $\mathbf{a}^{*}(x, \cdot), \mathbf{g}^{*}(x, \cdot)$ numerically.

Let $\mathcal{M}_{h}$ be a regular triangulation of $\Omega$ with mesh size $h$ and $\tau=T / N$ be the time step length, $t^{n}=n \tau, n=0,1, \cdots, N$. Further, let $W_{h}$ be the standard conforming linear finite element space over $\mathcal{M}_{h}$ and $W_{h}^{0}=W_{h} \cap H_{0}^{1}(\Omega)$. For any $K \in \mathcal{M}_{h}$, denote

$$
\langle\cdot\rangle_{K}=\frac{1}{|K|} \int_{K}(\cdot) d x
$$

as the volume average over $K$.

Set $v=b(u)$. For $n=1, \cdots, N$, our discrete problem is to seek $v_{h}^{n} \in W_{h}^{0}$, the approximate solution of $v$ at time $t=t^{n}$, such that

$$
\left(\frac{v_{h}^{n}-v_{h}^{n-1}}{\tau}, w_{h}\right)+\left(\tilde{\mathbf{g}}\left(x, \tilde{u}^{n}\right)+\tilde{\mathbf{a}}\left(x, \tilde{u}^{n}\right) \nabla \tilde{u}^{n}, \nabla w_{h}\right)=\left(\bar{f}^{n}, w_{h}\right) \quad \forall w_{h} \in W_{h}^{0}
$$

where $\tilde{u}^{n}=b^{-1}\left(v_{h}^{n}\right), v_{h}^{0}=b\left(u_{0}\right)$ and $\bar{f}^{n}=\tau^{-1} \int_{t^{n-1}}^{t^{n}} f(x, t) d t$. For any $s \in \mathbb{R}$, the nonlinear functions $\tilde{\mathbf{a}}(x, s)$ and $\tilde{\mathbf{g}}(x, s)$ are piecewise constant over $\mathcal{M}_{h}$ defined as follows.

For any $K \in \mathcal{M}_{h}, s \in \mathbb{R}$, let $p_{i}^{\varepsilon}, i=1,2, \cdots, d$, be the solution of the problem

$$
\begin{aligned}
-\nabla \cdot\left(\mathbf{a}^{\varepsilon}(x, s) \nabla p_{i}^{\varepsilon}\right) & =0 & \text { in } K \\
p_{i}^{\varepsilon} & =x_{i} & \text { on } \partial K
\end{aligned}
$$

Then, on $K, \tilde{\mathbf{a}}$ is a constant tensor determined by the following system

$$
\tilde{\mathbf{a}}\left\langle\nabla p_{i}^{\varepsilon}\right\rangle_{K}=\left\langle\mathbf{a}^{\varepsilon}(x, s) \nabla p_{i}^{\varepsilon}\right\rangle_{K}, \quad i=1,2, \cdots, d .
$$

It is well-defined since by using Green's formula

$$
\left\langle\nabla p_{i}^{\varepsilon}\right\rangle_{K}=\frac{1}{|K|} \int_{\partial K} x_{i} \mathbf{n} d \sigma=\mathbf{e}_{i}
$$

where $\mathbf{e}_{i}$ is the unit vector in the $i$ th direction. Similar to the argument in $[4,34]$ for the linear case, we know that $\tilde{\mathbf{a}}$ is symmetric, bounded, and positive definite. Moreover,

$$
\mathbf{e}_{i} \cdot\left(\tilde{\mathbf{a}} \mathbf{e}_{j}\right)=\left\langle\nabla p_{i}^{\varepsilon} \cdot\left(\mathbf{a}^{\varepsilon}(x, s) \nabla p_{j}^{\varepsilon}\right)\right\rangle_{K}
$$

Further, $\tilde{\mathbf{g}}(x, s)$ is a constant vector in $K$ determined by

$$
\tilde{g}_{i}(x, s)=\left\langle\mathbf{g}^{\varepsilon}(x, s) \cdot \nabla p_{i}^{\varepsilon}\right\rangle_{K}, \quad i=1,2, \cdots, d .
$$

It is easy to show $\left|\tilde{g}_{i}(x, s)\right| \leq C$.

The existence and uniqueness of the solution of problem (2.5) can be obtained by the standard method (see, e.g. [30, Chapter 13]). 


\section{Homogenization theory}

In this section, we assume that $\mathbf{a}^{\varepsilon}\left(x, u_{\varepsilon}\right)$ has the form $\mathbf{a}\left(u_{\varepsilon}, x / \varepsilon\right)$ and $\mathbf{g}^{\varepsilon}\left(x, u_{\varepsilon}\right)$ has the form $\mathbf{g}\left(u_{\varepsilon}, x / \varepsilon\right)$, where $a_{i j}(s, y), g_{i}(s, y) \in C^{1}\left(\mathbb{R} ; C_{p}^{1}\left(\mathbb{R}^{d}\right)\right)$. Here $C_{p}^{1}\left(\mathbb{R}^{d}\right)$ stands for the collection of all $C^{1}\left(\mathbb{R}^{d}\right)$ periodic functions with respect to the unit cube $Y$. It is shown that under these assumptions ( $\mathrm{cf}$. $[2,21,26]$ ), $u_{\varepsilon}$ converges in a suitable topology to the solution of the homogenized equation

$$
\begin{aligned}
\partial_{t} b(u)-\nabla \cdot\left(\mathbf{g}^{*}(u)+\mathbf{a}^{*}(u) \nabla u\right) & =f(x, t) & & \text { in } Q_{T}, \\
u & =0 & & \text { on } S_{T}, \\
u(x, 0) & =u_{0}(x) & & \text { in } \Omega,
\end{aligned}
$$

where

$$
a_{i j}^{*}(s)=\frac{1}{|Y|} \int_{Y} a_{i k}(s, y)\left(\delta_{k j}+\frac{\partial \chi^{j}}{\partial y_{k}}(s, y)\right) d y .
$$

$\chi^{j}(s, y)$ is the periodic solution of

$$
-\nabla_{y} \cdot\left(\mathbf{a}(s, y) \nabla_{y} \chi^{j}(s, y)\right)=\nabla_{y} \cdot\left(\mathbf{a}(s, y) \mathbf{e}_{j}\right), \quad j=1, \cdots, d
$$

with zero mean, i.e., $\int_{Y} \chi^{j} d y=0$, where $\mathbf{e}_{j}$ is the unit vector in the $j$ th direction. Further,

$$
g_{i}^{*}(s)=\frac{1}{|Y|} \int_{Y}\left(g_{i}(s, y)+a_{i k} \frac{\partial \eta}{\partial y_{k}}(s, y)\right) d y,
$$

where $\eta$ is the periodic solution of

$$
-\nabla_{y} \cdot\left(\mathbf{a}(s, y) \nabla_{y} \eta(s, y)\right)=\nabla_{y} \cdot(\mathbf{g}(s, y))
$$

with zero mean, i.e., $\int_{Y} \eta d y=0$.

Moreover, it is shown in [16, Lemma 2.6] that $\mathbf{a}^{*}(s)$ satisfies

$$
\lambda|\xi|^{2} \leq a_{i j}^{*}(s) \xi_{i} \xi_{j} \leq \Lambda|\xi|^{2} \quad \forall \xi \in \mathbb{R}^{d}, s \in \mathbb{R}
$$

with some positive constants $\lambda$ and $\Lambda$.

We remark that $s$ plays the role of a parameter in (3.2)-(3.5). Since $a_{i j}(s, y)$ and $g_{i}(s, y)$ are differentiable in $s$, we can easily show that $a_{i j}^{*}(s), g_{i}^{*}(s), \chi^{j}(s, y)$ and $\eta(s, y)$ are also differentiable in $s$. Moreover, $\chi^{j}, \eta \in C^{1}\left(\mathbb{R} ; C_{p}^{1}\left(\mathbb{R}^{d}\right)\right.$ ) (see [23]).

The variational problem of (3.1) is to seek $u(x, t) \in \mathcal{W}$, for almost every $t \in(0, T)$, $u(\cdot, t) \in H_{0}^{1}(\Omega)$ such that $u(x, 0)=u_{0}(x)$ in $\Omega$,

$$
\left(\partial_{t} b(u), v\right)+\left(\mathbf{g}^{*}(u)+\mathbf{a}^{*}(u) \nabla u, \nabla v\right)=(f, v) \quad \forall v \in H_{0}^{1}(\Omega) .
$$

In the following, we will always assume that

$$
u \in W_{2}^{2,1}\left(Q_{T}\right), \nabla u \in L^{\infty}\left(Q_{T}\right), \nabla u_{t} \in L^{2}\left(Q_{T}\right) .
$$

Set

$$
u_{1}(x, t, x / \varepsilon)=\chi^{j}(u, x / \varepsilon) \frac{\partial u}{\partial x_{j}}+\eta(u, x / \varepsilon),
$$


and denote $u_{1}^{\varepsilon}(x, t)=u(x, t)+\varepsilon u_{1}(x, t, x / \varepsilon)$. Let $\theta_{\varepsilon}$ be the boundary corrector

$$
\begin{aligned}
& b^{\prime}\left(u_{1}^{\varepsilon}+\varepsilon \theta_{\varepsilon}\right) \partial_{t} \theta_{\varepsilon}-\nabla \cdot\left(\mathbf{a}\left(u_{\varepsilon}, x / \varepsilon\right) \nabla \theta_{\varepsilon}\right)=0 \text { in } Q_{T}, \\
& \theta_{\varepsilon}=-u_{1}(x, t, x / \varepsilon) \text { on } S_{T}, \quad \theta_{\varepsilon}(x, 0)=-u_{1}(x, 0, x / \varepsilon) \text { in } \Omega .
\end{aligned}
$$

By the Maximum Principle, we have

$$
\left\|\theta_{\varepsilon}\right\|_{L^{\infty}\left(Q_{T}\right)} \leq C\left(\|\nabla u\|_{L^{\infty}\left(Q_{T}\right)}+\left\|u_{0}\right\|_{1, \infty, \Omega}\right) .
$$

Lemma 3.1. Assume that (3.7) holds. Then there exists a constant $C$ independent of $\varepsilon, \Omega$ such that

$$
\left\|\varepsilon \theta_{\varepsilon}\right\|_{L^{\infty}\left(0, T ; L^{2}(\Omega)\right)} \leq C \varepsilon, \quad\left\|\varepsilon \nabla \theta_{\varepsilon}\right\|_{L^{2}\left(Q_{T}\right)} \leq C \sqrt{\varepsilon} .
$$

Proof. The first inequality follows from (3.10). To show the second inequality, denote $\Omega_{\varepsilon}:=\{x$, dist $\{x, \partial \Omega\} \geq \varepsilon\}$. Let $\xi \in C_{0}^{\infty}(\Omega)$ be the cut-off function such that $0 \leq \xi \leq 1$ in $\mathbb{R}^{d}, \xi \equiv 1$ in $\Omega \backslash \Omega_{\varepsilon / 2}, \xi=0$ in $\Omega_{\varepsilon}$ and $|\nabla \xi| \leq \hat{C} / \varepsilon$ in $\Omega$ with $\hat{C}$ independent of $\varepsilon$ and $\Omega$. Define $d(x, t)=1 / b^{\prime}\left(u_{1}^{\varepsilon}+\varepsilon \theta_{\varepsilon}\right)$. Taking $\left(\theta_{\varepsilon}-\xi u_{1}(x, t, x / \varepsilon)\right) d(x, t)$ as the test function of (3.9) yields

$$
\begin{aligned}
\left(\partial_{t} \theta_{\varepsilon}, \theta_{\varepsilon}\right) & +\left(\mathbf{a}\left(u_{\varepsilon}, x / \varepsilon\right) \nabla \theta_{\varepsilon}, \nabla\left(d(x, t) \theta_{\varepsilon}\right)\right) \\
& =\left(\partial_{t} \theta_{\varepsilon}, \xi u_{1}\right)+\left(\mathbf{a}\left(u_{\varepsilon}, x / \varepsilon\right) \nabla \theta_{\varepsilon}, \nabla\left(d(x, t) \xi u_{1}\right)\right) .
\end{aligned}
$$

It is clear that

$$
\left(\mathbf{a}\left(u_{\varepsilon}, x / \varepsilon\right) \nabla \theta_{\varepsilon}, d(x, t) \nabla \theta_{\varepsilon}\right) \geq \frac{\lambda}{b_{2}}\left\|\nabla \theta_{\varepsilon}\right\|_{0, \Omega}^{2}
$$

A simple calculation shows

$$
|\nabla d(x, t)| \leq C\left|\nabla\left(u_{1}^{\varepsilon}+\varepsilon \theta_{\varepsilon}\right)\right| \leq C(1+|\nabla u|)+C \varepsilon\left(\left|D^{2} u\right|+\left|\nabla \theta_{\varepsilon}\right|\right) .
$$

Thus, we have

$$
\begin{aligned}
& \left|\left(\mathbf{a}\left(u_{\varepsilon}, x / \varepsilon\right) \nabla \theta_{\varepsilon}, \theta_{\varepsilon} \nabla d(x, t)\right)\right| \\
& \quad \leq C\left\|\nabla \theta_{\varepsilon}\right\|_{0, \Omega}\left(\left\|\theta_{\varepsilon}\right\|_{0, \Omega}+\varepsilon\left(\left\|D^{2} u\right\|_{0, \Omega}+\left\|\nabla \theta_{\varepsilon}\right\|_{0, \Omega}\right)\right) \\
& \quad \leq C \varepsilon\left\|\nabla \theta_{\varepsilon}\right\|_{0, \Omega}^{2}+\frac{\lambda}{8 b_{2}}\left\|\nabla \theta_{\varepsilon}\right\|_{0, \Omega}^{2}+C\|u\|_{2, \Omega}^{2}+C\left\|\theta_{\varepsilon}\right\|_{0, \Omega}^{2} .
\end{aligned}
$$

Here we have used the assumption (3.7) and (3.10). Further, it is easy to show that

$$
\begin{aligned}
\left\|\nabla\left(\xi u_{1}\right)\right\|_{0, \Omega} & =\left\|\nabla\left(\xi u_{1}\right)\right\|_{0, \Omega \backslash \Omega^{\varepsilon}} \\
& \leq C\left(1+\|\nabla u\|_{0, \infty, \Omega}\right) \sqrt{|\partial \Omega| \varepsilon / \varepsilon}+C\|u\|_{2, \Omega} \\
& \leq C \varepsilon^{-1 / 2}+C\|u\|_{2, \Omega}
\end{aligned}
$$

Hence

$$
\begin{aligned}
\left\|\nabla\left(d(x, t) \xi u_{1}\right)\right\|_{0, \Omega} \leq & \|\nabla d(x, t)\|_{0, \Omega}\left\|\xi u_{1}\right\|_{0, \infty, \Omega} \\
& +\|d(x, t)\|_{0, \infty, \Omega}\left\|\nabla\left(\xi u_{1}\right)\right\|_{0, \Omega} \\
\leq & C \varepsilon\left\|\nabla \theta_{\varepsilon}\right\|_{0, \Omega}+C\|u\|_{2, \Omega}+C \varepsilon^{-1 / 2}
\end{aligned}
$$


Then, we have

$$
\begin{aligned}
\left(\mathbf{a}\left(u_{\varepsilon}, x / \varepsilon\right) \nabla \theta_{\varepsilon}, \nabla\left(d(x, t) \xi u_{1}\right)\right) \leq & C \varepsilon\left\|\nabla \theta_{\varepsilon}\right\|_{0, \Omega}^{2}+\frac{\lambda}{8 b_{2}}\left\|\nabla \theta_{\varepsilon}\right\|_{0, \Omega}^{2} \\
& +C\|u\|_{2, \Omega}^{2}+C \varepsilon^{-1}
\end{aligned}
$$

Further, by using the Poincare inequality, integration by parts gives

$$
\begin{aligned}
\left|\int_{0}^{t}\left(\partial_{t} \theta_{\varepsilon}, \xi u_{1}\right) d s\right| & =\left|\left(\theta_{\varepsilon}, \xi u_{1}\right)\right|_{0}^{t}-\int_{0}^{t}\left(\theta_{\varepsilon}, \xi \partial_{t} u_{1}\right) d s \mid \\
& \leq C+C \int_{0}^{t}\left(\left\|\theta_{\varepsilon}\right\|_{0, \Omega}^{2}+\left\|\partial_{t} u_{1}\right\|_{0, \Omega}^{2}\right) d s
\end{aligned}
$$

Note here we have used (3.10) and $\left|\int_{\Omega} \theta_{\varepsilon}^{2}(x, 0) d x\right| \leq C\left\|\nabla u_{0}\right\|_{0, \Omega}^{2}$. By integrating (3.12) over $(0, t)$, it follows from $(3.14)-(3.18)$ that

$$
\int_{\Omega} \theta_{\varepsilon}^{2}(t) d x+\int_{0}^{t} \int_{\Omega}\left|\nabla \theta_{\varepsilon}\right|^{2} d x d s \leq C \int_{\Omega} \theta_{\varepsilon}^{2}(x, 0) d x+C \varepsilon^{-1}+C \int_{0}^{t}\left\|\theta_{\varepsilon}\right\|_{0, \Omega}^{2} d s .
$$

This completes the proof upon using the Gronwall inequality.

THEOREM 3.2. Let $u_{\varepsilon}$ and $u$ be the solutions of (2.1) and (3.1) respectively. Assume that (3.7) holds. Then there exists a constant $C$ independent of $\varepsilon, \Omega$ such that

$$
\left\|b\left(u_{\varepsilon}\right)-b\left(u_{1}^{\varepsilon}+\varepsilon \theta_{\varepsilon}\right)\right\|_{L^{\infty}\left(0, T ; L^{2}(\Omega)\right)}+\left\|\nabla\left(u_{\varepsilon}-\left(u_{1}^{\varepsilon}+\varepsilon \theta_{\varepsilon}\right)\right)\right\|_{L^{2}\left(Q_{T}\right)} \leq C \sqrt{\varepsilon} .
$$

Proof. By the simple calculations, we get

$$
\begin{aligned}
a_{i j}\left(u_{\varepsilon}, x / \varepsilon\right) & \frac{\partial\left(u+\varepsilon \chi^{k}(u, x / \varepsilon) \partial u / \partial x_{k}\right)}{\partial x_{j}}-a_{i j}^{*}(u) \frac{\partial u}{\partial x_{j}} \\
= & \left(a_{i j}\left(u_{\varepsilon}, x / \varepsilon\right)-a_{i j}(u, x / \varepsilon)\right)\left(\delta_{k j}+\frac{\partial \chi^{k}}{\partial y_{j}}(u, x / \varepsilon)\right) \frac{\partial u}{\partial x_{k}} \\
& +G_{i}^{k}(u, x / \varepsilon) \frac{\partial u}{\partial x_{k}}+\varepsilon a_{i j}\left(u_{\varepsilon}, x / \varepsilon\right) \chi^{k}(u, x / \varepsilon) \frac{\partial^{2} u}{\partial x_{j} \partial x_{k}} \\
& +\varepsilon a_{i j}\left(u_{\varepsilon}, x / \varepsilon\right) \frac{\partial \chi^{k}}{\partial s}(u, x / \varepsilon) \frac{\partial u}{\partial x_{k}} \frac{\partial u}{\partial x_{j}}
\end{aligned}
$$

where

$$
G_{i}^{k}(s, y)=a_{i j}(s, y)\left(\delta_{k j}+\frac{\partial \chi^{k}}{\partial y_{j}}(s, y)\right)-a_{i k}^{*}(s)
$$

From the definitions of $a_{i k}^{*}$ and $\chi^{k}$, it follows that

$$
\int_{Y} G_{i}^{k}(s, y) d y=0 \quad \text { and } \quad \frac{\partial G_{i}^{k}(s, y)}{\partial y_{i}}=0 .
$$

Then there exist skew-symmetric matrices (see $\left[22\right.$, p.6]) $\alpha^{k}(s, y)=\left(\alpha_{i j}^{k}(s, y)\right)$ such that

$$
G_{i}^{k}(s, y)=\frac{\partial}{\partial y_{l}}\left(\alpha_{l i}^{k}(s, y)\right), \quad \int_{Y} \alpha_{l i}^{k}(s, y) d y=0
$$


With this notation, we can rewrite

$$
\begin{aligned}
G_{i}^{k}(u, x / \varepsilon) \frac{\partial u}{\partial x_{k}}= & \varepsilon \frac{\partial}{\partial x_{l}}\left(\alpha_{l i}^{k}(u, x / \varepsilon) \frac{\partial u}{\partial x_{k}}\right)-\varepsilon \alpha_{l i}^{k}(u, x / \varepsilon) \frac{\partial^{2} u}{\partial x_{l} \partial x_{k}} \\
& -\varepsilon \frac{\partial \alpha_{l i}^{k}}{\partial s}(u, x / \varepsilon) \frac{\partial u}{\partial x_{l}} \frac{\partial u}{\partial x_{k}} .
\end{aligned}
$$

On the other hand, by the simple calculations, we get

$$
\begin{aligned}
a_{i j}\left(u_{\varepsilon}, x / \varepsilon\right) & \frac{\varepsilon \partial \eta(u, x / \varepsilon)}{\partial x_{j}}+g_{i}\left(u_{\varepsilon}, x / \varepsilon\right)-g_{i}^{*}(u) \\
= & \left(a_{i j}\left(u_{\varepsilon}, x / \varepsilon\right)-a_{i j}(u, x / \varepsilon)\right) \frac{\partial \eta}{\partial y_{j}}(u, x / \varepsilon) \\
& +\varepsilon a_{i j}\left(u_{\varepsilon}, x / \varepsilon\right) \frac{\partial \eta}{\partial s}(u, x / \varepsilon) \frac{\partial u}{\partial x_{j}}+g_{i}\left(u_{\varepsilon}, x / \varepsilon\right)-g_{i}(u, x / \varepsilon) \\
& +H_{i}(u, x / \varepsilon),
\end{aligned}
$$

where

$$
H_{i}(s, y)=g_{i}(s, y)+a_{i j}(s, y) \frac{\partial \eta}{\partial y_{j}}(s, y)-g_{i}^{*}(s)
$$

From the definitions of $g_{i}^{*}$ and $\eta$, it follows that

$$
\int_{Y} H_{i}(s, y) d y=0 \quad \text { and } \quad \frac{\partial H_{i}(s, y)}{\partial y_{i}}=0 .
$$

Then there exists a skew-symmetric matrix $\left(\right.$ see $[22$, p.6] $) \beta(s, y)=\left(\beta_{i j}(s, y)\right)$ such that

$$
H_{i}(s, y)=\frac{\partial}{\partial y_{l}}\left(\beta_{l i}(s, y)\right), \quad \int_{Y} \beta_{l i}(s, y) d y=0 .
$$

With this notation, we can rewrite

$$
H_{i}(u, x / \varepsilon)=\varepsilon \frac{\partial}{\partial x_{l}}\left(\beta_{l i}(u, x / \varepsilon)\right)-\varepsilon \frac{\partial \beta_{l i}}{\partial s}(u, x / \varepsilon) \frac{\partial u}{\partial x_{l}} .
$$

From (2.4) and (3.6), for any $v \in H_{0}^{1}(\Omega)$, we have

$$
\left(\partial_{t}\left(b\left(u_{\varepsilon}\right)-b(u)\right), v\right)+\left(\mathbf{a}\left(u_{\varepsilon}, x / \varepsilon\right) \nabla u_{\varepsilon}+\mathbf{g}\left(u_{\varepsilon}, x / \varepsilon\right)-\mathbf{a}^{*}(u) \nabla u-\mathbf{g}^{*}(u), \nabla v\right)=0 .
$$

Thus, in view of (3.20) and (3.21), we obtain

$$
\begin{aligned}
\left(\partial _ { t } \left(b\left(u_{\varepsilon}\right)-\right.\right. & \left.\left.b\left(u_{1}^{\varepsilon}+\varepsilon \theta_{\varepsilon}\right)\right), v\right)+\left(\mathbf{a}\left(u_{\varepsilon}, x / \varepsilon\right) \nabla\left(u_{\varepsilon}-\left(u_{1}^{\varepsilon}+\varepsilon \theta_{\varepsilon}\right)\right), \nabla v\right) \\
= & -\left(b^{\prime}\left(u_{1}^{\varepsilon}+\varepsilon \theta_{\varepsilon}\right) \partial_{t}\left(u+\varepsilon u_{1}\right)-b^{\prime}(u) \partial_{t} u, v\right) \\
& -\left(\mathbf{R}_{1}, \nabla v\right)-\left(\mathbf{R}_{2}, \nabla v\right)-\varepsilon\left(\zeta_{i}, \partial v / \partial x_{i}\right) \\
:= & \mathrm{I}_{1}+\cdots+\mathrm{I}_{4},
\end{aligned}
$$

where

$$
R_{1}^{i}=\left(a_{i j}\left(u_{\varepsilon}, x / \varepsilon\right)-a_{i j}(u, x / \varepsilon)\right)\left[\left(\delta_{k j}+\frac{\partial \chi^{k}}{\partial y_{j}}(u, x / \varepsilon)\right) \frac{\partial u}{\partial x_{k}}+\frac{\partial \eta}{\partial y_{j}}(u, x / \varepsilon)\right],
$$


and

$$
R_{2}^{i}=g_{i}\left(u_{\varepsilon}, x / \varepsilon\right)-g_{i}(u, x / \varepsilon),
$$

and

$$
\begin{gathered}
\zeta_{i}=a_{i j}\left(u_{\varepsilon}, x / \varepsilon\right)\left(\chi^{k}(u, x / \varepsilon) \frac{\partial^{2} u}{\partial x_{j} \partial x_{k}}+\frac{\partial \chi^{k}}{\partial s}(u, x / \varepsilon) \frac{\partial u}{\partial x_{j}} \frac{\partial u}{\partial x_{k}}+\frac{\partial \eta}{\partial s}(u, x / \varepsilon) \frac{\partial u}{\partial x_{j}}\right) \\
+\alpha_{i j}^{k}(u, x / \varepsilon) \frac{\partial^{2} u}{\partial x_{j} \partial x_{k}}+\frac{\partial \alpha_{i j}^{k}}{\partial s}(u, x / \varepsilon) \frac{\partial u}{\partial x_{j}} \frac{\partial u}{\partial x_{k}}+\frac{\partial \beta_{i j}}{\partial s}(u, x / \varepsilon) \frac{\partial u}{\partial x_{j}} .
\end{gathered}
$$

Notice here we have used the fact $\frac{\partial}{\partial x_{j}}\left(\alpha_{i j}^{k}(u, x / \varepsilon) \frac{\partial u}{\partial x_{k}}\right)$ and $\frac{\partial}{\partial x_{j}}\left(\beta_{i j}(u, x / \varepsilon)\right)$ are divergence free.

Set $v=b\left(u_{\varepsilon}\right)-b\left(u_{1}^{\varepsilon}+\varepsilon \theta_{\varepsilon}\right)$. It is obvious that

$$
\nabla v=b^{\prime}\left(u_{\varepsilon}\right) \nabla\left(u_{\varepsilon}-\left(u_{1}^{\varepsilon}+\varepsilon \theta_{\varepsilon}\right)\right)+\left(b^{\prime}\left(u_{\varepsilon}\right)-b^{\prime}\left(u_{1}^{\varepsilon}+\varepsilon \theta_{\varepsilon}\right)\right) \nabla\left(u_{1}^{\varepsilon}+\varepsilon \theta_{\varepsilon}\right),
$$

and

$$
\begin{aligned}
\left(b^{\prime}\left(u_{\varepsilon}\right)-\right. & \left.b^{\prime}\left(u_{1}^{\varepsilon}+\varepsilon \theta_{\varepsilon}\right)\right) \frac{\partial\left(u_{1}^{\varepsilon}+\varepsilon \theta_{\varepsilon}\right)}{\partial x_{i}} \\
= & \left(b^{\prime}\left(u_{\varepsilon}\right)-b^{\prime}\left(u_{1}^{\varepsilon}+\varepsilon \theta_{\varepsilon}\right)\right)\left(\frac{\partial u}{\partial x_{i}}+\frac{\partial \chi^{j}}{\partial y_{i}} \frac{\partial u}{\partial x_{j}}+\frac{\partial \eta}{\partial y_{i}}\right) \\
+ & \varepsilon\left(b^{\prime}\left(u_{\varepsilon}\right)-b^{\prime}\left(u_{1}^{\varepsilon}+\varepsilon \theta_{\varepsilon}\right)\right)\left(\chi^{j} \frac{\partial^{2} u}{\partial x_{j} \partial x_{i}}\right. \\
+ & \left.\frac{\partial \chi^{j}}{\partial s} \frac{\partial u}{\partial x_{i}} \frac{\partial u}{\partial x_{j}}+\frac{\partial \eta}{\partial s} \frac{\partial u}{\partial x_{i}}+\frac{\partial \theta_{\varepsilon}}{\partial x_{i}}\right) .
\end{aligned}
$$

Hence, in view of (2.3) and by use of the coercive condition and Young's inequality, we obtain

$$
\begin{aligned}
& \left(\mathbf{a}\left(u_{\varepsilon}, x / \varepsilon\right) \nabla\left(u_{\varepsilon}-\left(u_{1}^{\varepsilon}+\varepsilon \theta_{\varepsilon}\right)\right), \nabla v\right) \\
& \quad \geq \frac{\lambda b_{1}}{2}\left\|\nabla\left(u_{\varepsilon}-\left(u_{1}^{\varepsilon}+\varepsilon \theta_{\varepsilon}\right)\right)\right\|_{0, \Omega}^{2}-C \varepsilon^{2}\left(\|u\|_{2, \Omega}^{2}+\left\|\nabla \theta_{\varepsilon}\right\|_{0, \Omega}^{2}\right) \\
& \quad-C\left\|b\left(u_{\varepsilon}\right)-b\left(u_{1}^{\varepsilon}+\varepsilon \theta_{\varepsilon}\right)\right\|_{0, \Omega}^{2},
\end{aligned}
$$

and

$$
\begin{aligned}
\|\nabla v\|_{0, \Omega}^{2} \leq & C\left\|\nabla\left(u_{\varepsilon}-\left(u_{1}^{\varepsilon}+\varepsilon \theta_{\varepsilon}\right)\right)\right\|_{0, \Omega}^{2}+C \varepsilon^{2}\left(\|u\|_{2, \Omega}^{2}+\left\|\nabla \theta_{\varepsilon}\right\|_{0, \Omega}^{2}\right) \\
& +C\left\|b\left(u_{\varepsilon}\right)-b\left(u_{1}^{\varepsilon}+\varepsilon \theta_{\varepsilon}\right)\right\|_{0, \Omega}^{2} .
\end{aligned}
$$

Now we give the estimates of the terms $\mathrm{I}_{1}, \cdots, \mathrm{I}_{4}$. It is clear that

$$
\begin{aligned}
\left|\mathrm{I}_{1}\right| & \left.=\mid \varepsilon\left(b^{\prime}\left(u_{1}^{\varepsilon}+\varepsilon \theta_{\varepsilon}\right) \partial_{t} u_{1}, v\right)+\left(b^{\prime}\left(u_{1}^{\varepsilon}+\varepsilon \theta_{\varepsilon}\right)-b^{\prime}(u)\right) \partial_{t} u, v\right) \mid \\
& \leq C \varepsilon\left(\left\|\partial_{t} u_{1}\right\|_{0, \Omega}+\left\|u_{1}+\theta_{\varepsilon}\right\|_{0, \infty, \Omega}\left\|\partial_{t} u\right\|_{0, \Omega}\right)\|v\|_{0, \Omega} \\
& \leq C \varepsilon^{2}\left(\left\|\nabla u_{t}\right\|_{0, \Omega}^{2}+\left\|u_{t}\right\|_{0, \Omega}^{2}\right)+C\left\|b\left(u_{\varepsilon}\right)-b\left(u_{1}^{\varepsilon}+\varepsilon \theta_{\varepsilon}\right)\right\|_{0, \Omega}^{2},
\end{aligned}
$$

and

$$
\left|\mathrm{I}_{4}\right| \leq C \varepsilon^{2}\left(\|u\|_{2, \Omega}^{2}+1\right)+C\|\nabla v\|_{0, \Omega}^{2} .
$$


Further, since

$$
\begin{aligned}
& \left|a_{i j}\left(u_{\varepsilon}, x / \varepsilon\right)-a_{i j}(u, x / \varepsilon)\right| \\
& \quad \leq\left|a_{i j}\left(u_{\varepsilon}, x / \varepsilon\right)-a_{i j}\left(u_{1}^{\varepsilon}+\varepsilon \theta_{\varepsilon}, x / \varepsilon\right)\right|+\left|a_{i j}\left(u_{1}^{\varepsilon}+\varepsilon \theta_{\varepsilon}, x / \varepsilon\right)-a_{i j}(u, x / \varepsilon)\right| \\
& \quad \leq C\left|b\left(u_{\varepsilon}\right)-b\left(u_{1}^{\varepsilon}+\varepsilon \theta_{\varepsilon}\right)\right|+C \varepsilon\left|u_{1}+\theta_{\varepsilon}\right|,
\end{aligned}
$$

it follows that

$$
\left|\mathrm{I}_{2}\right| \leq C \varepsilon^{2}\left(\|u\|_{1, \Omega}^{2}+\left\|\theta_{\varepsilon}\right\|_{0, \Omega}^{2}\right)+C\left\|b\left(u_{\varepsilon}\right)-b\left(u_{1}^{\varepsilon}+\varepsilon \theta_{\varepsilon}\right)\right\|_{0, \Omega}^{2}+C\|\nabla v\|_{0, \Omega}^{2} .
$$

Similarly, we can show that

$$
\left|\mathrm{I}_{3}\right| \leq C \varepsilon^{2}\left(\|u\|_{1, \Omega}^{2}+\left\|\theta_{\varepsilon}\right\|_{0, \Omega}^{2}\right)+C\left\|b\left(u_{\varepsilon}\right)-b\left(u_{1}^{\varepsilon}+\varepsilon \theta_{\varepsilon}\right)\right\|_{0, \Omega}^{2}+C\|\nabla v\|_{0, \Omega}^{2} .
$$

Finally, integrating $(3.22)$ over $(0, t)$, it follows from $(3.25)-(3.30)$ that

$$
\begin{gathered}
\frac{1}{2} \int_{\Omega}\left(b\left(u_{\varepsilon}\right)-b\left(u_{1}^{\varepsilon}+\varepsilon \theta_{\varepsilon}\right)\right)^{2} d x+\frac{\lambda b_{1}}{8} \int_{0}^{t} \int_{\Omega}\left|\nabla\left(u_{\varepsilon}-\left(u_{1}^{\varepsilon}+\varepsilon \theta_{\varepsilon}\right)\right)\right|^{2} d x d s \\
\leq C \varepsilon^{2}+C \varepsilon^{2} \int_{0}^{t}\left\|\nabla \theta_{\varepsilon}\right\|_{0, \Omega}^{2} d s+C \int_{0}^{t}\left\|b\left(u_{\varepsilon}\right)-b\left(u_{1}^{\varepsilon}+\varepsilon \theta_{\varepsilon}\right)\right\|_{0, \Omega}^{2} d s
\end{gathered}
$$

Note here we have used $\left\|b\left(u_{0}\right)-b\left(u_{0}+\varepsilon(\xi-1) u_{1}(x, 0)\right)\right\|_{0, \Omega} \leq C \varepsilon\left\|\nabla u_{0}\right\|_{0, \Omega}$. Thus, in view of Lemma 3.1, by using Gronwall's inequality we complete the proof.

Since we assume that $b^{\prime}(s) \geq b_{1}>0$, the following theorem follows from Theorem 3.2 and Lemma 3.1 .

THEOREM 3.3. Let $u_{\varepsilon}$ and $u$ be the solutions of (2.1) and (3.1) respectively. Assume that (3.7) holds. Then there exists a constant $C$ independent of $\varepsilon, \Omega$ such that

$$
\left\|u_{\varepsilon}-u-\varepsilon u_{1}\right\|_{L^{\infty}\left(0, T ; L^{2}(\Omega)\right)}+\left\|\nabla\left(u_{\varepsilon}-u-\varepsilon u_{1}\right)\right\|_{L^{2}\left(Q_{T}\right)} \leq C \sqrt{\varepsilon} .
$$

\section{Error estimate for the periodic medium}

We now study the accuracy of the upscaling method given in Section 2. Note here we assume that $\mathbf{a}^{\varepsilon}$ and $\mathbf{g}^{\varepsilon}$ have the periodic forms.

For the local problem (2.6), it is easy to check that

$$
p_{i}^{\varepsilon}=x_{i}+\varepsilon \chi^{i}(s, x / \varepsilon)-\varepsilon \theta_{i}^{\varepsilon},
$$

where $\chi^{i}(s, y)$ is defined by (3.3) and $\theta_{i}^{\varepsilon}$ is the solution of

$$
\begin{aligned}
-\nabla \cdot\left(\mathbf{a}(s, x / \varepsilon) \nabla \theta_{i}^{\varepsilon}\right) & =0 & & \text { in } K, \\
\theta_{i}^{\varepsilon} & =\chi^{i}(s, x / \varepsilon) & & \text { on } \partial K .
\end{aligned}
$$

It is obvious that

$$
\left\|\nabla p_{i}^{\varepsilon}\right\|_{0, K} \leq C\left\|\mathbf{e}_{i}\right\|_{0, K} \leq C h^{d / 2},
$$

and, for $\theta_{i}^{\varepsilon}$ we have (cf. [20] or [5, Theorem 3.1])

$$
\left\|\theta_{i}^{\varepsilon}\right\|_{0, K} \leq C h^{d / 2}, \quad\left\|\nabla \theta_{i}^{\varepsilon}\right\|_{0, K} \leq C h^{(d-1) / 2} \varepsilon^{-1 / 2} .
$$


Lemma 4.1. Given domain $K$ with diam $(K)=h$, let $\Phi(s, y)$ defined in $Y$ be a $Y$ periodic function in $y$, where $Y$ is a unit cube and $s \in \mathbb{R}$ is fixed. Furthermore, assume $\|\Phi(s, y)\|_{L^{\infty}(Y)} \leq \hat{C}$, where $\hat{C}$ is independent of $h, \varepsilon$ and $s$. Then, we have

$$
\left|\frac{1}{|Y|} \int_{Y} \Phi(s, y) d y-\langle\Phi(s, x / \varepsilon)\rangle_{K}\right| \leq C \varepsilon h^{-1},
$$

where $C$ is independent of $\varepsilon, h$ and $s$.

Proof. Let $Y_{i} \subset K$ be a rescaled $Y$ of size $\varepsilon$. Denote $K^{\prime}=\cup_{K_{i} \subset K} Y_{i}$. We have

$$
\begin{aligned}
& \left|\frac{1}{|Y|} \int_{Y} \Phi(s, y) d y-\langle\Phi(s, x / \varepsilon)\rangle_{K}\right| \\
& \quad \leq\left|\frac{1}{|Y|} \int_{Y} \Phi(s, y) d y-\frac{\varepsilon}{|K|} \sum_{Y_{i} \subset K^{\prime}} \int_{Y} \Phi(s, y) d y\right|+\left|\frac{1}{|K|} \int_{K \backslash K^{\prime}} \Phi(s, x / \varepsilon) d x\right| \\
& \quad \leq C \frac{|K|-\left|K^{\prime}\right|}{|K|} \leq C \varepsilon h^{-1} .
\end{aligned}
$$

Note here we have used $\sum_{Y_{i} \subset K^{\prime}}=\left|K^{\prime}\right| /(\varepsilon|Y|)$.

Lemma 4.2. In each $K \in \mathcal{M}_{h}$, for any $s \in \mathbb{R}$, we have

$$
\left|a_{i j}^{*}(s)-\tilde{a}_{i j}(s)\right| \leq C \varepsilon h^{-1},
$$

where $C$ is independent of $\varepsilon, h$ and $s$.

Proof. Similar proof can be found in [34]. For the convenience of the reader, we sketch the proof here. Denote $\mathbf{w}_{i}=\mathbf{e}_{i}+\nabla_{y} \chi^{i}(s, y)$. By the integration by parts, it follows from (3.2) and (3.3) that

$$
a_{i j}^{*}(s)=\frac{1}{|Y|} \int_{Y} \mathbf{w}_{i} \cdot\left(\mathbf{a}(s, y) \mathbf{w}_{j}\right) d y,
$$

and

$$
\nabla_{y} \cdot\left(\mathbf{a}(s, y) \mathbf{w}_{j}\right)=0 .
$$

It follows from (4.1) that $\nabla p_{i}^{\varepsilon}=\mathbf{w}_{i}-\varepsilon \nabla \theta_{i}^{\varepsilon}$. Hence, from (2.9), we have

$$
\begin{aligned}
a_{i j}^{*}(s)-\tilde{a}_{i j}(s)= & \left(\frac{1}{|Y|} \int_{Y} \mathbf{w}_{i} \cdot\left(\mathbf{a}(s, y) \mathbf{w}_{j}\right) d y-\left\langle\mathbf{w}_{i} \cdot\left(\mathbf{a}(s, x / \varepsilon) \mathbf{w}_{j}\right)\right\rangle_{K}\right) \\
& +\varepsilon\left\langle\mathbf{w}_{i} \cdot\left(\mathbf{a}(s, x / \varepsilon) \nabla \theta_{j}^{\varepsilon}\right)\right\rangle_{K}+\varepsilon\left\langle\nabla \theta_{i}^{\varepsilon} \cdot\left(\mathbf{a}(s, x / \varepsilon) \mathbf{w}_{j}\right)\right\rangle_{K} \\
& -\varepsilon^{2}\left\langle\nabla \theta_{i}^{\varepsilon} \cdot\left(\mathbf{a}(s, x / \varepsilon) \nabla \theta_{j}^{\varepsilon}\right)\right\rangle_{K} \\
:= & \mathrm{II}_{1}+\mathrm{II}_{2}+\mathrm{II}_{3}+\mathrm{II}_{4} .
\end{aligned}
$$

By Lemma 4.1, we have $\left|\mathrm{II}_{1}\right| \leq C \varepsilon h^{-1}$. Further, by (4.4) and the Cauchy-Schwartz inequality, we also have $\left|\mathrm{II}_{4}\right| \leq C \varepsilon h^{-1}$. For the term $\mathrm{II}_{3}$, since $\mathbf{a}(s, x / \varepsilon) \mathbf{w}_{j}$ is divergence free, integration by parts gives

$$
\begin{aligned}
\left|\int_{K} \nabla \theta_{i}^{\varepsilon} \cdot\left(\mathbf{a}(s, x / \varepsilon) \mathbf{w}_{j}\right) d x\right| & =\left|\int_{\partial K} \theta_{i}^{\varepsilon} \mathbf{n} \cdot\left(\mathbf{a}(s, x / \varepsilon) \mathbf{w}_{j}\right) d \sigma\right| \\
& =\left|\int_{\partial K}\left(\chi^{i} \mathbf{n} \cdot\left(\mathbf{a w}_{j}\right)\right)(s, x / \varepsilon) d \sigma\right| \leq C h^{d-1} .
\end{aligned}
$$


$\mathrm{II}_{2}$ can be estimated similarly since $\mathbf{a}$ is symmetric. The proof now is completed.

Let $\phi^{\varepsilon}$ be the solution of the problem

$$
\begin{aligned}
-\nabla \cdot\left(\mathbf{a}(s, x / \varepsilon) \nabla \phi^{\varepsilon}\right) & =\nabla \cdot \mathbf{g}(s, x / \varepsilon) & & \text { in } K, \\
\phi^{\varepsilon} & =0 & & \text { on } \partial K .
\end{aligned}
$$

For problem (4.10), it is easy to check that

$$
\phi^{\varepsilon}=\varepsilon \eta(s, x / \varepsilon)-\varepsilon \theta_{\eta}^{\varepsilon},
$$

where $\eta(s, y)$ is defined by $(3.5)$ and $\theta_{\eta}^{\varepsilon}$ is the solution of

$$
\begin{aligned}
\nabla \cdot\left(\mathbf{a}(s, x / \varepsilon) \nabla \theta_{\eta}^{\varepsilon}\right) & =0 & & \text { in } K, \\
\theta_{\eta}^{\varepsilon} & =\eta(s, x / \varepsilon) & & \text { on } \partial K .
\end{aligned}
$$

It is obvious that

$$
\left\|\nabla \theta_{\eta}^{\varepsilon}\right\|_{0, K} \leq C h^{(d-1) / 2} \varepsilon^{-1 / 2} .
$$

To estimate the error between $\tilde{\mathbf{g}}$ and $\mathbf{g}^{*}$, we need the following lemma (see also [14]).

LEMMA 4.3. We have

$$
g_{i}^{*}(s)=\frac{1}{|Y|} \int_{Y} g_{k}(s, y)\left(\delta_{i k}+\frac{\partial \chi^{i}}{\partial y_{k}}(s, y)\right) d y, \quad i=1, \cdots, d .
$$

Proof. Choosing $\chi^{j}$ as the test function in the variational formula of (3.5), we obtain

$$
\begin{aligned}
-\left(\mathbf{g}(s, y), \nabla_{y} \chi^{j}(s, y)\right)_{Y} & =\left(\mathbf{a}(s, y) \nabla_{y} \eta(s, y), \nabla_{y} \chi^{j}(s, y)\right)_{Y} \\
& =-\left(\mathbf{a}(s, y) \mathbf{e}_{j}, \nabla_{y} \eta(s, y)\right)_{Y} .
\end{aligned}
$$

Note in the second step here that we have used the variational formula of (3.3) with $\eta(s, y)$ as the test function. Thus, we have

$$
\int_{Y} \mathbf{g}(s, y) \cdot \nabla_{y} \chi^{j}(s, y) d y=\int_{Y} \mathbf{a}(s, y) \nabla_{y} \eta(s, y) \cdot \mathbf{e}_{j} d y
$$

which follows the results from (3.4) immediately.

LEMMA 4.4. In each $K \in \mathcal{M}_{h}$, for any $s \in \mathbb{R}$, we have

$$
\left|g_{i}^{*}(s)-\tilde{g}_{i}(s)\right| \leq C \varepsilon h^{-1},
$$

where $C$ is independent of $\varepsilon, h$ and $s$.

Proof. We still denote $\mathbf{w}_{i}=\mathbf{e}_{i}+\nabla_{y} \chi^{i}(s, y)$. From Lemma 4.3, we have

$$
g_{i}^{*}(s)=\frac{1}{|Y|} \int_{Y} \mathbf{g}(s, y) \cdot \mathbf{w}_{i} d y .
$$

Hence, it follows from $\nabla p_{i}^{\varepsilon}=\mathbf{w}_{i}-\varepsilon \nabla \theta_{i}^{\varepsilon}$ that

$$
\begin{aligned}
g_{i}^{*}(s)-\tilde{g}_{i}(s)= & \left(\frac{1}{|Y|} \int_{Y} \mathbf{g}(s, y) \cdot \mathbf{w}_{i} d y-\left\langle\mathbf{g}(s, x / \varepsilon) \cdot \mathbf{w}_{i}\right\rangle_{K}\right) \\
& +\varepsilon\left\langle\mathbf{g}(s, x / \varepsilon) \cdot \nabla \theta_{i}^{\varepsilon}\right\rangle_{K} .
\end{aligned}
$$


By Lemma 4.1, we know that the first part of r.h.s of (4.18) is bounded by $C \varepsilon h^{-1}$. For the second part, in view of that fact $\int_{K} \mathbf{a} \nabla \phi^{\varepsilon} \cdot \nabla \theta_{i}^{\varepsilon} d x=0$ and $\nabla \phi^{\varepsilon}=\nabla_{y} \eta(x / \varepsilon)-\varepsilon \nabla \theta_{\eta}^{\varepsilon}$, we have

$$
\begin{aligned}
\int_{K} \mathbf{g}(s, x / \varepsilon) \cdot \nabla \theta_{i}^{\varepsilon} d x= & \int_{K}\left(\mathbf{g}+\mathbf{a} \nabla_{y} \eta\right)(s, x / \varepsilon) \cdot \nabla \theta_{i}^{\varepsilon} d x \\
& -\varepsilon \int_{K} \mathbf{a}(s, x / \varepsilon) \nabla \theta_{\eta}^{\varepsilon} \cdot \nabla \theta_{i}^{\varepsilon} d x \\
:= & \mathrm{III}_{1}+\mathrm{III}_{2} .
\end{aligned}
$$

From (4.4), (4.13) and the Cauchy-Schwartz inequality, we have $\left|\mathrm{III}_{2}\right| \leq C h^{d-1}$. For the term $\mathrm{III}_{1}$, in view of $\nabla_{y} \cdot\left(\mathbf{g}+\mathbf{a} \nabla_{y} \eta\right)=0$, integration by parts gives

$$
\begin{aligned}
\left|\int_{K}\left(\mathbf{g}+\mathbf{a} \nabla_{y} \eta\right)(s, x / \varepsilon) \cdot \nabla \theta_{i}^{\varepsilon} d x\right| & =\left|\int_{\partial K} \theta_{i}^{\varepsilon} \mathbf{n} \cdot\left(\mathbf{g}+\mathbf{a} \nabla_{y} \eta\right)(s, x / \varepsilon) d \sigma\right| \\
& \leq C h^{d-1} .
\end{aligned}
$$

Thus, we obtain $\left|\varepsilon\left\langle\mathbf{g}(s, x / \varepsilon) \cdot \nabla \theta_{i}^{\varepsilon}\right\rangle_{K}\right| \leq C \varepsilon h^{-1}$. The proof now is completed.

THEOREM 4.5. For $n=1, \cdots, N$, let $u^{n}$ and $v_{h}^{n}$ be the solution of (3.6) and (2.5) respectively. Denote $v^{n}=b\left(u^{n}\right)$. Then

$$
\begin{aligned}
\left\|v^{m}-v_{h}^{m}\right\|_{0, \Omega}+ & \left(\sum_{1}^{m} \tau\left\|\nabla\left(v^{n}-v_{h}^{n}\right)\right\|_{0, \Omega}^{2}\right)^{1 / 2} \\
& \leq C(u)\left(\tau+h+\frac{\varepsilon}{h}\right) \quad \forall m=1, \cdots, N,
\end{aligned}
$$

where $C(u)$ depends on $\|u\|_{W_{2}^{1,2}\left(Q_{T}\right)},\left\|\nabla u_{t}\right\|_{L^{2}\left(Q_{T}\right)},\|\nabla u\|_{L^{\infty}\left(Q_{T}\right)}$, but not on $h, \tau$ and $\varepsilon$.

Proof. Setting $v=b(u)$ in (3.6) and integrating from $t^{n-1}$ to $t^{n}$ yields

$$
\left(\frac{v^{n}-v^{n-1}}{\tau}, w\right)+\left(\frac{1}{\tau} \int_{t^{n-1}}^{t^{n}}\left(\mathbf{g}^{*}(u)+\mathbf{a}^{*}(u) \nabla u\right) d t, \nabla w\right)=\left(\bar{f}^{n}, w\right) .
$$

Denote $\delta^{n}=I_{h} v^{n}-v_{h}^{n}$, where $I_{h}: H_{0}^{1}(\Omega) \rightarrow W_{h}^{0}$ is the usual Clemént interpolation operator, which satisfies the following estimates $([7])$

$$
\begin{aligned}
& \left\|v-I_{h} v\right\|_{H^{j}(\Omega)} \leq C h^{m-j}\|v\|_{H^{m}(\Omega)} \\
& \quad \forall v \in H^{m}(\Omega) \cap H_{0}^{1}(\Omega), \quad j=0,1, \cdots, m, \quad m=1,2 .
\end{aligned}
$$

Subtracting (2.5) from (4.21) yields

$$
\begin{aligned}
&\left(\frac{\delta^{n}-\delta^{n-1}}{\tau}, w_{h}\right)+\left(\frac{\tilde{\mathbf{a}}\left(\tilde{u}^{n}\right)}{b^{\prime}\left(\tilde{u}^{n}\right)} \nabla \delta^{n}, \nabla w_{h}\right) \\
&=-\left(\frac{v^{n}-v^{n-1}}{\tau}-\frac{I_{h} v^{n}-I_{h} v^{n-1}}{\tau}, w_{h}\right) \\
&-\left(\frac{1}{\tau} \int_{t^{n-1}}^{t^{n}} \frac{\mathbf{a}^{*}(u)}{b^{\prime}(u)} \nabla v d t-\frac{\tilde{\mathbf{a}}\left(\tilde{u}^{n}\right)}{b^{\prime}\left(\tilde{u}^{n}\right)} \nabla I_{h} v^{n}, \nabla w_{h}\right) \\
&-\left(\frac{1}{\tau} \int_{t^{n-1}}^{t^{n}} \mathbf{g}^{*}(u) d t-\tilde{\mathbf{g}}\left(\tilde{u}^{n}\right), \nabla w_{h}\right) \\
&:= \mathrm{IV}_{1}+\cdots+\mathrm{IV}_{3} .
\end{aligned}
$$


Recall that $\tilde{u}^{n}=b^{-1}\left(v_{h}^{n}\right)$. Set $w_{h}=\tau \delta^{n}$. It is clear that

$$
\begin{aligned}
\left|\mathrm{IV}_{1}\right| & \leq\left|\int_{\Omega} \int_{t^{n-1}}^{t^{n}}\left(\partial_{t} b(u)-I_{h} \partial_{t} b(u)\right) d t \delta^{n} d x\right| \\
& \leq C h^{2} \int_{t^{n-1}}^{t^{n}}\left\|\nabla u_{t}\right\|_{0, \Omega}^{2} d t+C \tau\left\|\delta^{n}\right\|_{0, \Omega}^{2} .
\end{aligned}
$$

Furthermore, it is obvious that

$$
\begin{aligned}
\mathrm{IV}_{2}= & -\left(\int_{t^{n-1}}^{t^{n}}\left(\frac{\mathbf{a}^{*}(u)}{b^{\prime}(u)}-\frac{\tilde{\mathbf{a}}\left(\tilde{u}^{n}\right)}{b^{\prime}\left(\tilde{u}^{n}\right)}\right) \nabla v d t, \nabla \delta^{n}\right) \\
& -\left(\int_{t^{n-1}}^{t^{n}} \frac{\tilde{\mathbf{a}}\left(\tilde{u}^{n}\right)}{b^{\prime}\left(\tilde{u}^{n}\right)} \nabla\left(v-I_{h} v^{n}\right) d t, \nabla \delta^{n}\right) .
\end{aligned}
$$

By Lemma 4.2 and the Lipschitz continuity of $\mathbf{a}^{*}$ and $b^{\prime}$, we know that

$$
\begin{aligned}
& \left|\frac{\mathbf{a}^{*}(u)}{b^{\prime}(u)}-\frac{\tilde{\mathbf{a}}\left(\tilde{u}^{n}\right)}{b^{\prime}\left(\tilde{u}^{n}\right)}\right| \\
& \quad \leq \frac{\left|\mathbf{a}^{*}(u)-\mathbf{a}^{*}\left(\tilde{u}^{n}\right)\right|}{\left|b^{\prime}(u)\right|}+\frac{\left|\mathbf{a}^{*}\left(\tilde{u}^{n}\right)-\tilde{\mathbf{a}}\left(\tilde{u}^{n}\right)\right|}{\left|b^{\prime}(u)\right|}+\left|\frac{1}{b^{\prime}(u)}-\frac{1}{b^{\prime}\left(\tilde{u}^{n}\right)}\right|\left|\tilde{\mathbf{a}}\left(\tilde{u}^{n}\right)\right| \\
& \quad \leq C\left|u-\tilde{u}^{n}\right|+C\left(h+\varepsilon h^{-1}\right) \\
& \quad=C\left|b^{-1}(v)-b^{-1}\left(v_{h}^{n}\right)\right|+C\left(h+\varepsilon h^{-1}\right) \\
& \quad \leq C\left|v-\tilde{v}^{n}\right|+C\left(h+\varepsilon h^{-1}\right) .
\end{aligned}
$$

Noticing that $\|\nabla v\|_{L^{\infty}} \leq C\|\nabla b(u)\|_{L^{\infty}} \leq C$, we obtain

$$
\begin{aligned}
\left|\mathrm{IV}_{2}\right| \leq C & \int_{t^{n-1}}^{t^{n}}\left(\left\|v-v_{h}^{n}\right\|_{0, \Omega}+\left\|\nabla\left(v-I_{h} v^{n}\right)\right\|_{0, \Omega}+h+\varepsilon h^{-1}\right) d t\left\|\nabla \delta^{n}\right\|_{0, \Omega} \\
\leq & \frac{\lambda}{4} \tau\left\|\nabla \delta^{n}\right\|_{0, \Omega}^{2}+C \tau\left\|\delta^{n}\right\|_{0, \Omega}^{2}+C \tau\left(h+\varepsilon h^{-1}\right)^{2} \\
& \quad+C\left(h^{2}+\tau^{2}\right) \int_{t^{n-1}}^{t^{n}}\left(\|u\|_{2, \Omega}^{2}+\left\|\nabla u_{t}\right\|_{0, \Omega}^{2}+\left\|u_{t}\right\|_{0, \Omega}^{2}\right) d t .
\end{aligned}
$$

Similarly, we have

$$
\begin{aligned}
\left|\mathrm{IV}_{3}\right| \leq \frac{\lambda}{4} & \tau\left\|\nabla \delta^{n}\right\|_{0, \Omega}^{2}+C \tau\left\|\delta^{n}\right\|_{0, \Omega}^{2}+C \tau\left(h+\varepsilon h^{-1}\right)^{2} \\
& +C\left(h^{2}+\tau^{2}\right) \int_{t^{n-1}}^{t^{n}}\left(\left\|u_{t}\right\|_{0, \Omega}^{2}+\left\|\nabla u^{n}\right\|_{0, \Omega}^{2}\right) d t
\end{aligned}
$$

Utilizing the above estimations and summing over $n$ from 1 to $m$ yield

$$
\begin{aligned}
\left\|\delta^{m}\right\|_{0, \Omega}^{2} & +\sum_{1}^{m} \tau\left\|\nabla \delta^{n}\right\|_{0, \Omega}^{2} \\
& \leq c(u)\left(h+\tau+\varepsilon h^{-1}\right)^{2}+c \tau \sum_{1}^{m}\left\|\delta^{n}\right\|_{0, \Omega}^{2},
\end{aligned}
$$


where $c(u)$ depends on $\|u\|_{W_{2}^{1,2}\left(Q_{T}\right)},\left\|\nabla u_{t}\right\|_{L^{2}\left(Q_{T}\right)},\|\nabla u\|_{L^{\infty}\left(Q_{T}\right)}$. Thus, if $\tau$ is small enough, by using the Gronwall inequality we obtain

$$
\left\|\delta^{m}\right\|_{0, \Omega}^{2}+\sum_{1}^{m} \tau\left\|\nabla \delta^{n}\right\|_{0, \Omega}^{2} \leq C\left(\tau+h+\varepsilon h^{-1}\right)^{2},
$$

where $C$ depends on $T, c(u)$, not on $h, \tau$ and $\varepsilon$. Finally, we obtain the result by the standard interpolation theory and the triangle inequality.

Theorem 4.6. For $n=1, \cdots, N$, let $u^{n}$ and $v_{h}^{n}$ be the solution of (3.6) and (2.5) respectively. Set $\tilde{u}^{n}=b^{-1}\left(v_{h}^{n}\right)$. Then

$$
\begin{gathered}
\left\|u^{m}-\tilde{u}^{m}\right\|_{0, \Omega}+\left(\sum_{1}^{m} \tau\left\|\nabla\left(u^{n}-\tilde{u}^{n}\right)\right\|_{0, \Omega}^{2}\right)^{1 / 2} \\
\leq C(u)\left(\tau+h+\frac{\varepsilon}{h}\right) \quad \forall m=1, \cdots, N,
\end{gathered}
$$

where $C(u)$ depends on $\|u\|_{W_{2}^{1,2}\left(Q_{T}\right)},\left\|\nabla u_{t}\right\|_{L^{2}\left(Q_{T}\right)},\|\nabla u\|_{L^{\infty}\left(Q_{T}\right)}$, not on $h, \tau$ and $\varepsilon$.

Proof. The result can be obtained by the following facts:

$$
\left\|u^{m}-\tilde{u}^{m}\right\|_{0, \Omega}=\left\|b^{-1}\left(v^{m}\right)-b^{-1}\left(v_{h}^{m}\right)\right\|_{0, \Omega} \leq C\left\|v^{m}-v_{h}^{m}\right\|_{0, \Omega}
$$

and

$$
\begin{aligned}
\| \nabla & \left(u^{n}-\tilde{u}^{n}\right)\left\|_{0, \Omega}=\right\| \nabla\left(u^{n}-b^{-1}\left(v_{h}^{n}\right)\right) \|_{0, \Omega} \\
& \leq C\left\|\left(\left(b^{-1}\right)^{\prime}\left(v^{n}\right)-\left(b^{-1}\right)^{\prime}\left(v_{h}^{n}\right)\right) \nabla v^{n}\right\|_{0, \Omega}+\left\|\left(b^{-1}\right)^{\prime}\left(v_{h}^{n}\right) \nabla\left(v^{n}-v_{h}^{n}\right)\right\|_{0, \Omega} \\
& \leq C\left\|v^{n}-v_{h}^{n}\right\|_{1, \Omega} .
\end{aligned}
$$

This completes the proof.

\section{Numerical results}

In this section, we present several numerical examples carried out for periodic and random log-normal permeabilities to demonstrate the ability of the coarse-grid models presented in Section 2. The coarse-grid models are compared with the fine-scale model solved on a fine mesh. We have employed the linear approximation scheme in [29] to solve the fine-scale equations.

From the assumptions, it follows that the problems considered in our paper satisfy the maximum principle. So, the range of unknown can be obtained by the initial value and boundary values. For example, the unknown of the example in this Section lies between 0 and -10 . In our method, we compute the effective constitutive relations in each coarse mesh element for the discrete values in the range. It seems very expensive, but it is a pre-processing procedure which can be done completely parallel. Note that the effective parameters can also be computed on the fly as in [11], [13] (i.e. the effective parameters are computed whenever they are needed) instead of being done in advance. This is the approach used in this paper to avoid the expensive computation to build up a database for the effective parameters. We emphasize again that the real significance of the upscaling method lies in its ability to solve the problems in coarse meshes. This is particularly advantageous when multiple simulations or realizations are necessary due to changes of boundary conditions or source functions for certain given fine micro-structures of the highly heterogeneous permeability of the porous media. In practical applications, one may build up the database of the effective parameters completely parallel in advance or on the fly. 
5.1. Exponential model with periodic coefficients. Consider the following Richards equation,

$$
\partial_{t} \theta(u)-\nabla \cdot\left(K(x, u) \nabla\left(u+x_{3}\right)\right)=0
$$

where $x_{3}$ denotes the vertical coordinate in the medium. For the exponential model (see [31] or [15] for more details), we have $\theta(u)=\theta_{s} e^{\beta u}, K(x, u)=K_{s}(x) e^{\alpha(x) u}$. Choosing $\theta_{s}=1, \beta=0.1$. The heterogeneity comes from $K_{s}(x)$ and $\alpha(x)$.

For the convenience of the reader, we sketch the linear approximation scheme here. The time discretization is based on the backward Euler method. The concept behind the linear approximation is a clever relaxation scheme for solving a nonlinear elliptic BVP at each time point of the time partitioning.

Let us denote the time step $\tau>0$ and the space $V:=\left\{v \in H^{1}(\Omega):\left.v\right|_{\Gamma_{\text {top }}}=\right.$ $\left.0,\left.v\right|_{\Gamma_{\text {bottom }}}=0\right\}$. The approximate solution $u_{i} \approx u\left(t_{i}\right)$ at a given time point $t_{i}=i \tau$ is obtained in an iteration process with respect to the relaxation parameter $\kappa$. The linearized scheme for a fixed $i \in\{1, \cdots, n\}$ and running $\kappa=1,2, \cdots$ reads as

$$
\begin{aligned}
u_{i, 0}= & u_{i-1} \\
\frac{L}{\tau}\left(u_{i, \kappa}, \varphi\right)+\left(K\left(u_{i-1}\right) \nabla u_{i, \kappa}, \nabla \varphi\right)= & -\left(K\left(u_{i-1}\right) \mathbf{e}_{3}, \nabla \varphi\right)+\frac{L}{\tau}\left(u_{i, \kappa-1}, \varphi\right) \\
& -\frac{1}{\tau}\left(\theta\left(u_{i, \kappa-1}\right), \varphi\right)+\frac{1}{\tau}\left(\theta\left(u_{i-1}\right), \varphi\right)
\end{aligned}
$$

for all $\varphi \in V$. Here $L$ is the Lipschitz constant of the function $\theta$ and $\mathbf{e}_{2}=(0,1)^{T}$. The iteration process stops when the following condition is satisfied

$$
\left\|u_{i, \kappa}-u_{i, \kappa-1}\right\|_{0, \Omega} \leq C_{d} \tau^{d},
$$

where $C_{d}>0, d>1$ are fixed constants. After stopping the iterations at $\kappa=\kappa_{i, \text { last }}$, we denote $u_{i}:=u_{\kappa_{i, \text { last }}}$ and switch to the next step.

First, we solve (5.1) in the domain $\Omega=(0,1) \times(0,1)$, and assume that $K_{s}(x)$ and $\alpha(x)$ have the following periodic form

$$
K^{\varepsilon}\left(x_{1}, x_{3}\right)=1 /\left[2+1.8 \sin \left(2 \pi\left(2 x_{3}-x_{1}\right) / \varepsilon\right)\right] / 117.4,
$$

and

$$
\alpha^{\varepsilon}\left(x_{1}, x_{3}\right)=1 /\left[2+1.8 \sin \left(2 \pi\left(2 x_{3}-x_{1}\right) / \varepsilon\right)\right] / 11.74 .
$$

Here we fix $\varepsilon=1 / 16$. Thus, we have $\overline{K_{s}}=0.01$, and $\bar{\alpha}=0.1$.

The corresponding boundary conditions read as follows:

$$
\begin{aligned}
& u=0 \text { on } \Gamma_{\text {top }}, \quad u=-10 \text { on } \Gamma_{\text {bottom }}, \\
& -K(x, u) \nabla\left(u+x_{3}\right) \cdot \mathbf{n}=0 \text { on } \Gamma_{\mathrm{LR}},
\end{aligned}
$$

where $\Gamma_{\text {top }}=\left\{\left(x_{1}, x_{3}\right): x_{1} \in(0,1), x_{3}=1\right\}, \Gamma_{\text {bottom }}=\left\{\left(x_{1}, x_{3}\right): x_{1} \in(0,1), x_{3}=0\right\}$, and $\Gamma_{\mathrm{LR}}=\left\{\left(x_{1}, x_{3}\right): x_{1}=0\right.$ or $\left.1, x_{3} \in(0,1)\right\}$. We impose the following initial condition

$$
u(x, 0)=-10 \text { in } \Omega .
$$

To demonstrate the effectiveness of our upscaling method, we solve (5.1), (5.4), and (5.5) in a $256 \times 256$ mesh. The obtained solution $u_{h}^{\varepsilon}$ is considered as the finescale solution to compare with the upscaled solution. The coarse-grid model uses $8 \times 8,16 \times 16,32 \times 32$ mesh respectively. 

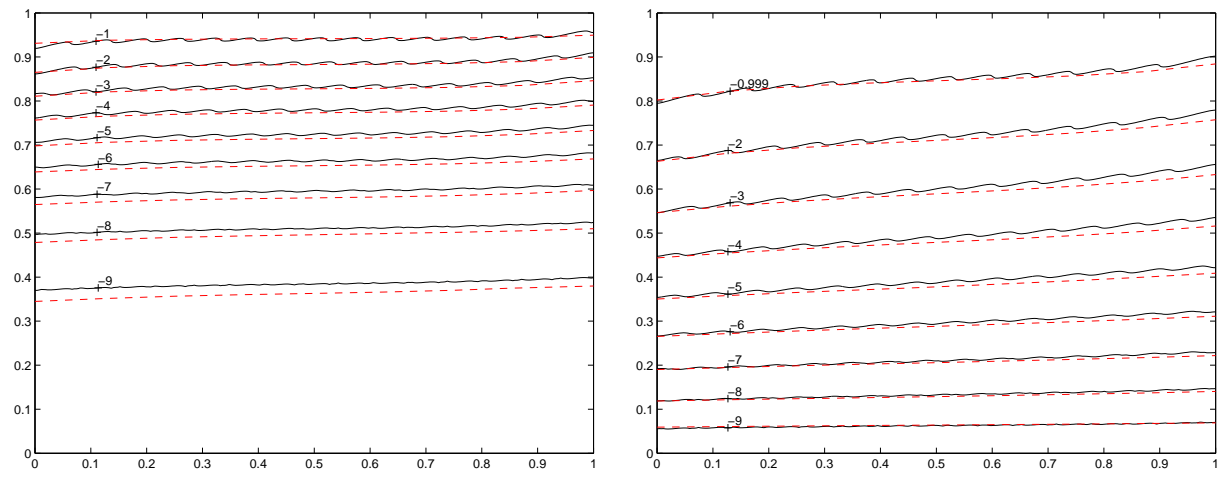

FIG. 5.1. The contour plots of $u_{h}^{\varepsilon}$ and $\tilde{u}_{h}$ at $t=1$ (left) and $t=10$ (right). The solid line stands for $u_{h}^{\varepsilon}$ and the dash line stands for $\tilde{u}_{h}$ in mesh $8 \times 8$. Periodic case $(\varepsilon=1 / 16)$.
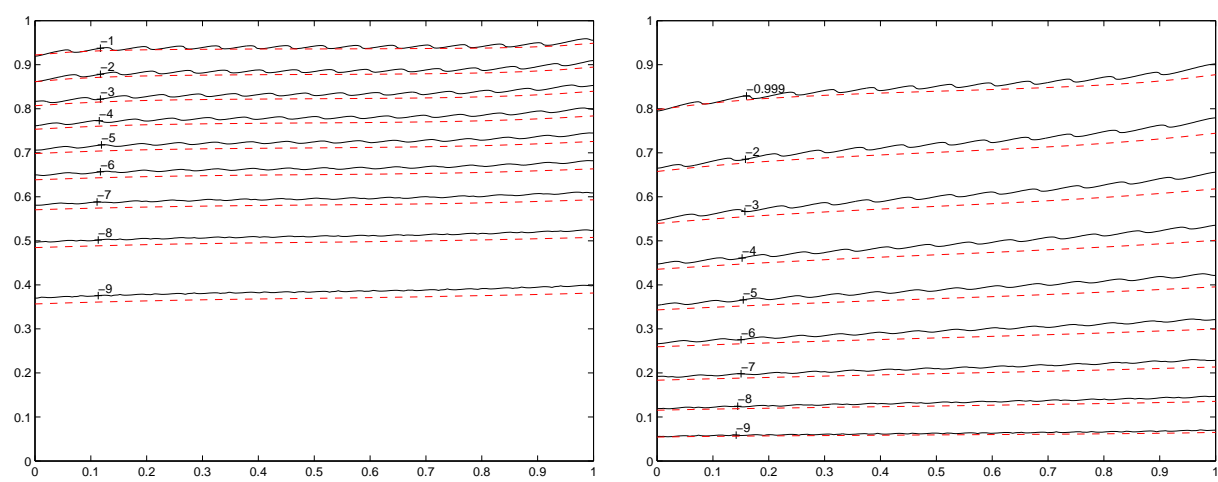

FIG. 5.2. The contour plots of $u_{h}^{\varepsilon}$ and $\tilde{u}_{h}$ at $t=1$ (left) and $t=10$ (right). The solid line stands for $u_{h}^{\varepsilon}$ and the dash line stands for $\tilde{u}_{h}$ in mesh $16 \times 16$. Periodic case $(\varepsilon=1 / 16)$.
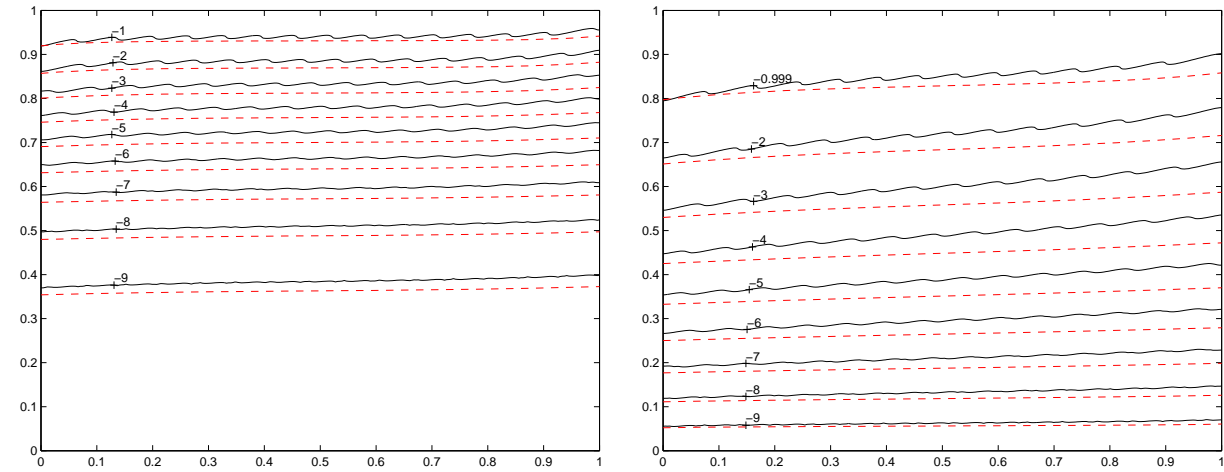

FIG. 5.3. The contour plots of $u_{h}^{\varepsilon}$ and $\tilde{u}_{h}$ at $t=1$ (left) and $t=10$ (right). The solid line stands for $u_{h}^{\varepsilon}$ and the dash line stands for $\tilde{u}_{h}$ in mesh $32 \times 32$. Periodic case $(\varepsilon=1 / 16)$. 

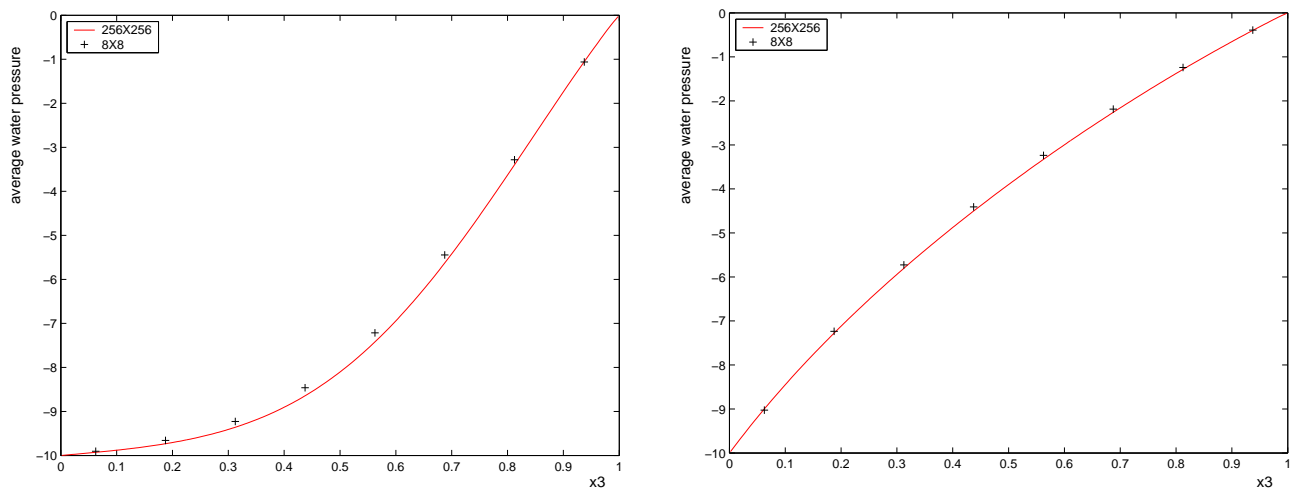

FIG. 5.4. The average solutions over the horizontal direction at $t=1$ (left) and $t=10$ (right). The solid line stands for the averaged $u_{h}^{\varepsilon}$ in a mesh $256 \times 256$, the + line stands for the averaged $\tilde{u}_{h}$ in a mesh $8 \times 8$. Periodic case $(\varepsilon=1 / 16)$.
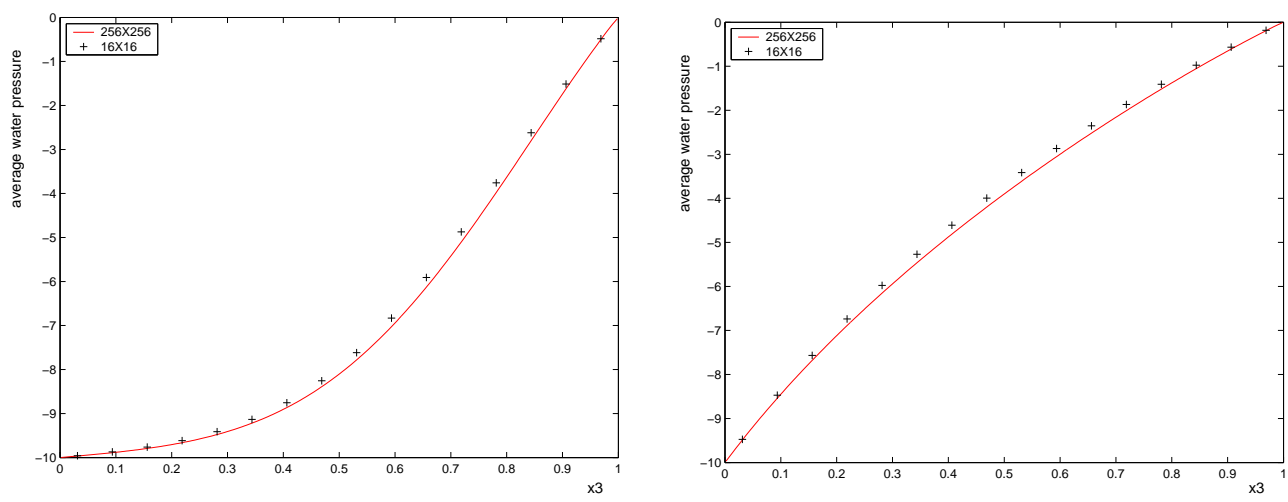

FIG. 5.5. The average solutions over the horizontal direction at $t=1$ (left) and $t=10$ (right). The solid line stands for the averaged $u_{h}^{\varepsilon}$ in a mesh $256 \times 256$, the + line stands for the averaged $\tilde{u}_{h}$ in a mesh $16 \times 16$. Periodic case $(\varepsilon=1 / 16)$.
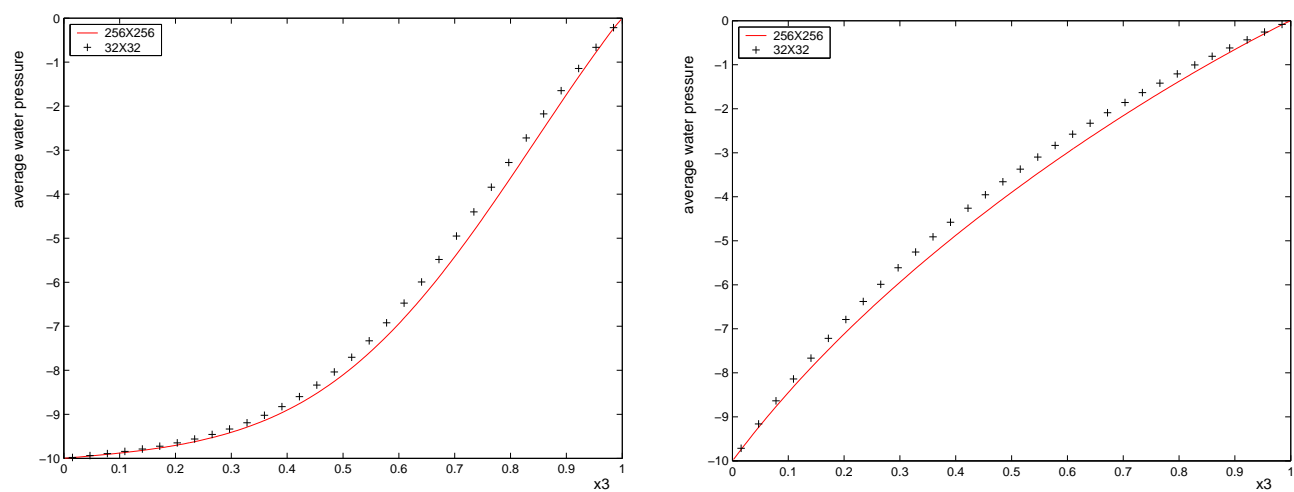

FIG. 5.6. The average solutions over the horizontal direction at $t=1$ (left) and $t=10$ (right). The solid line stands for the averaged $u_{h}^{\varepsilon}$ in a mesh $256 \times 256$, the + line stands for the averaged $\tilde{u}_{h}$ in a mesh $32 \times 32$. Periodic case $(\varepsilon=1 / 16)$. 
The contour plots of the water pressure $\tilde{u}_{h}$ (corresponding $u_{h}^{\varepsilon}$ ) at times $t=1$ and $t=10$ are depicted in Figures 5.1-5.3 for the coarse $8 \times 8,16 \times 16,32 \times 32$ mesh respectively. We observe that the upscaled solution on the $8 \times 8$ mesh provides the best agreement with the fine-scale solution. This may be explained by the effect of resonance error (the term $\varepsilon / h$ ) in the error estimate in Theorem 4.6.

We note that the problems that we have considered are vertical infiltration on the porous medium. Hence, it is also useful to compare the averaged water pressure which is obtained by taking an average over the horizontal direction $\left(x_{1}\right.$-axis $)$. The averaged pressure head curves are depicted in Figure 5.4-5.6 for the coarse $8 \times 8,16 \times 16,32 \times 32$ mesh respectively. The averaged pressure head is plotted against the depth $x_{3}$. It gives excellent agreement between the coarse mesh and fine mesh calculations.

5.2. Exponential model with random coefficients. It has been observed that the distribution of the hydraulic conductivity $K_{s}$ and the model parameter $\alpha$ are log-normal (see $[28,33])$. Thus, in this subsection we consider the applications of our upscaling method to Richards equation with randomly generated log-normal permeabilities.

For the exponential model, we choose $\theta_{s}=1$ and the same boundary conditions and initial condition as that of periodic case. The heterogeneity comes from $K_{s}(x)$ and $\alpha(x)$. We generate the random log-normal permeability fields $K_{s}(x)$ by using the moving ellipse average technique [8] with the variance of the logarithm of the permeability $\sigma=1.5$, and the correlation lengths $l_{1}=l_{3}=0.1$ (isotropic heterogeneities) in $x_{1}$ and $x_{3}$ directions, respectively. We let $\overline{K_{s}}=0.01$, and $\alpha(x)=10 K_{s}(x)$ hence $\bar{\alpha}=0.1$ in the realizations. One realization of the resulting permeability field in our numerical experiments is depicted in Figure 5.7. We also generate the random log-normal permeability field $K_{s}(x)$ (see Figure 5.8 for one realization) with the correlation lengths $l_{1}=0.1$ and $l_{3}=0.01$ respectively, which represents the the anisotropic heterogeneity. We let $\overline{K_{s}}=0.1$, and $\alpha(x)=0.5 K_{s}(x)$ hence $\bar{\alpha}=0.05$ in the realizations. We also choose $\beta=0.1$ and $\beta=0.05$ for the isotropic and anisotropic cases respectively. The fine-scale equation is solved in a $256 \times 256$ mesh, while the coarse-grid model uses a $16 \times 16$ mesh.
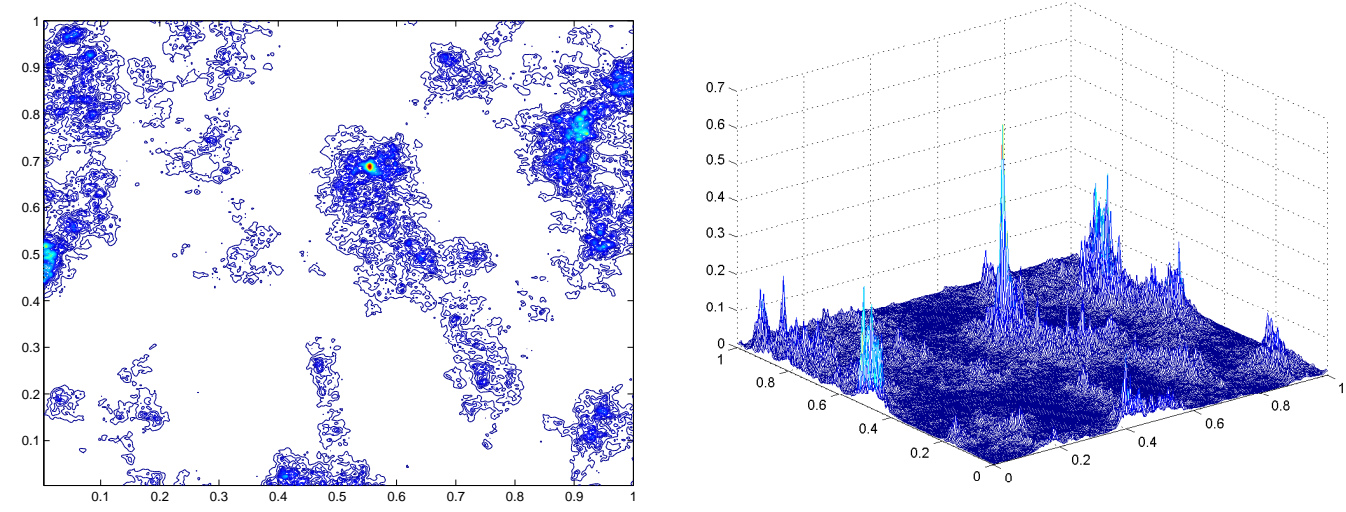

FIG. 5.7. The random log-normal permeability field $K_{s}(x)$. The ratio of maximum to minimum is $4.1323 E+04$. Case $l_{1}=l_{3}=0.1$. 

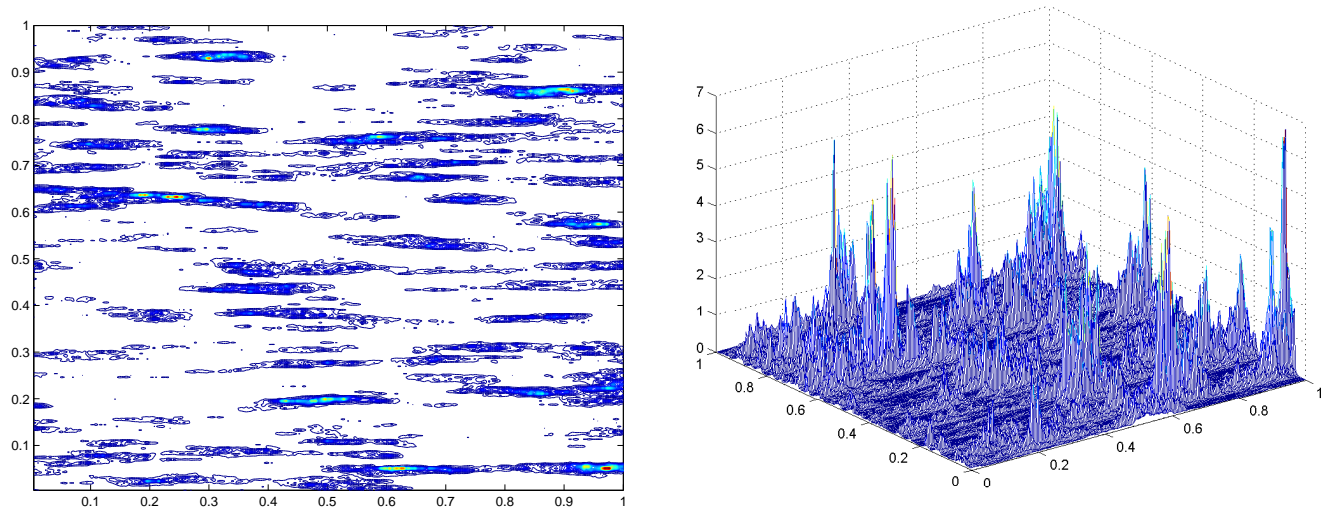

FIG. 5.8. The random log-normal permeability field $K_{s}(x)$. The ratio of maximum to minimum is $2.9704 E+04$. Case $l_{1}=0.1, l_{3}=0.01$.

The contour plots of the water pressure $\tilde{u}_{h}$ (corresponding $u_{h}^{\varepsilon}$ ) at time $t=1$ and $t=10$ are depicted in Figure 5.9 for the isotropic heterogeneity. We observe that the upscaled solution captures the macro-behavior effectively.

The contour plots of the water pressure $\tilde{u}_{h}$ (corresponding $u_{h}^{\varepsilon}$ ) at time $t=0.5$, $t=1, t=6$ and $t=10$ are depicted in Figure 5.10 and Figure 5.11 for the anisotropic heterogeneity. We observe that the upscaled solution does not match the macrobehavior so well as the isotropic case. One possible way to improve the accuracy of the upscaling method is to use the over-sampling technique in [12] developed for linear problems to compute the effective parameters. This is an interesting topic which deserves further investigation.

We also compare the averaged water pressure which is obtained by taking an average over the horizontal direction $\left(x_{1}\right.$-axis). The averaged pressure head curves are depicted in Figures 5.12-5.14 for the isotropic and anisotropic heterogeneities respectively. It gives excellent agreement between the coarse mesh and fine mesh calculations also.
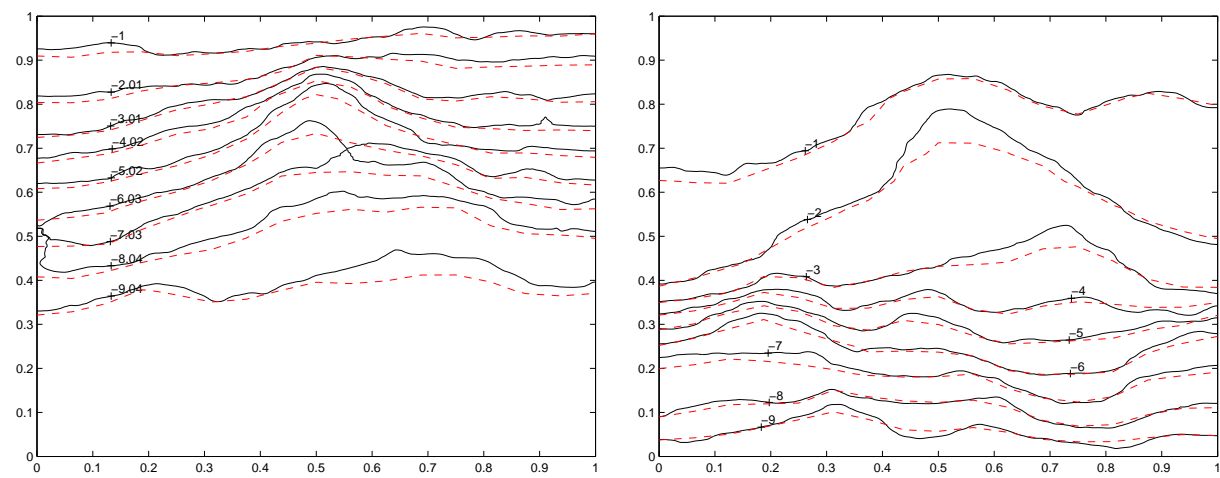

FIG. 5.9. The contour plots of $u_{h}^{\varepsilon}$ and $\tilde{u}_{h}$ at time $t=1$ (left) and $t=10$ (right). The solid line stands for $u_{h}^{\varepsilon}$ and the dash line stands for $\tilde{u}_{h}$ in a mesh $16 \times 16$. Case $l_{1}=l_{3}=0.1, \sigma=1.5$. 

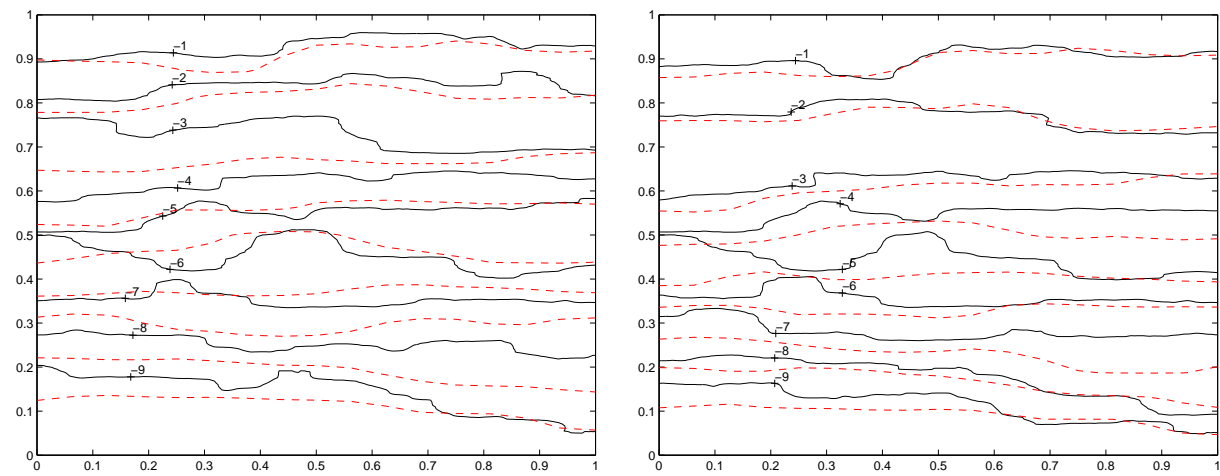

FIG. 5.10. The contour plots of $u_{h}^{\varepsilon}$ and $\tilde{u}_{h}$ at time $t=0.5$ (left) and $t=1$ (right). The solid line stands for $u_{h}^{\varepsilon}$ and the dash line stands for $\tilde{u}_{h}$ in a mesh $16 \times 16$. Case $l_{1}=0.1, l_{3}=0.01, \sigma=1.5$.
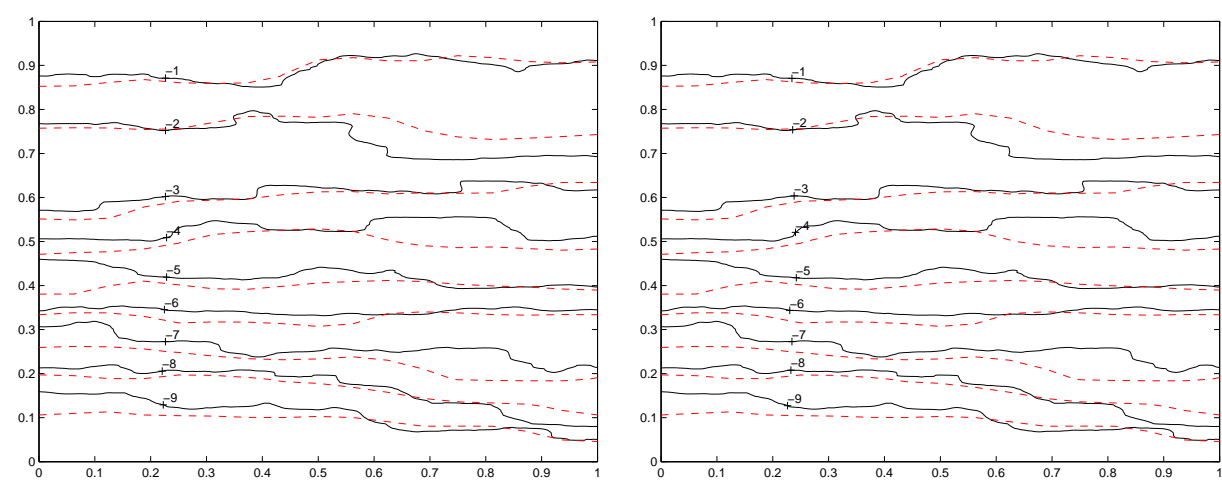

FIG. 5.11. The contour plots of $u_{h}^{\varepsilon}$ and $\tilde{u}_{h}$ at time $t=6$ (left) and $t=10$ (right). The solid line stands for $u_{h}^{\varepsilon}$ and the dash line stands for $\tilde{u}_{h}$ in a mesh $16 \times 16$. Case $l_{1}=0.1, l_{3}=0.01, \sigma=1.5$.
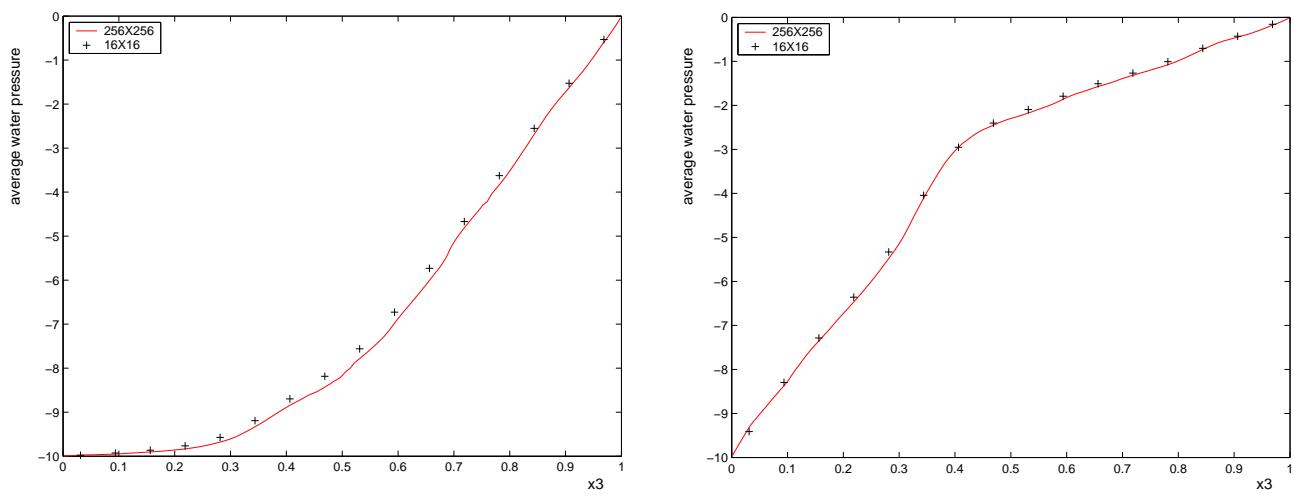

FIG. 5.12. The average solution over the horizontal direction at $t=1$ (left) and $t=10$ (right). The solid line stands for the averaged $u_{h}^{\varepsilon}$ in a mesh $256 \times 256$, the + line stands for the averaged $\tilde{u}_{h}$ in a mesh $16 \times 16$. Case $l_{1}=l_{3}=0.1, \sigma=1.5$. 

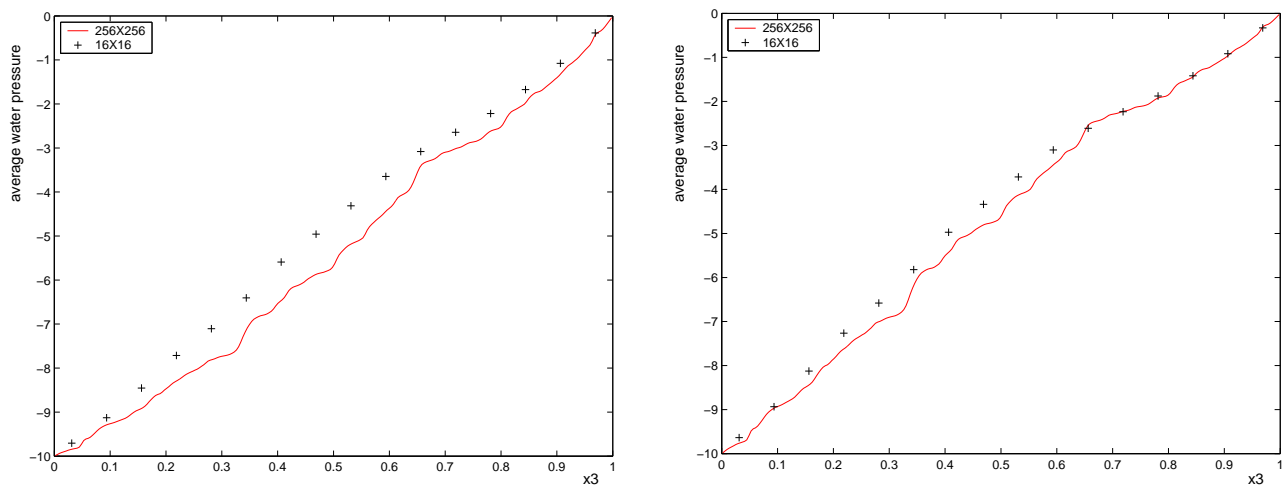

FIG. 5.13. The average solution over the horizontal direction at time $t=0.5$ (left) and $t=1$ (right). The solid line stands for the averaged $u_{h}^{\varepsilon}$ in a mesh $256 \times 256$, the + line stands for the averaged $\tilde{u}_{h}$ in a mesh $16 \times 16$. Case $l_{1}=0.1, l_{3}=0.01, \sigma=1.5$.
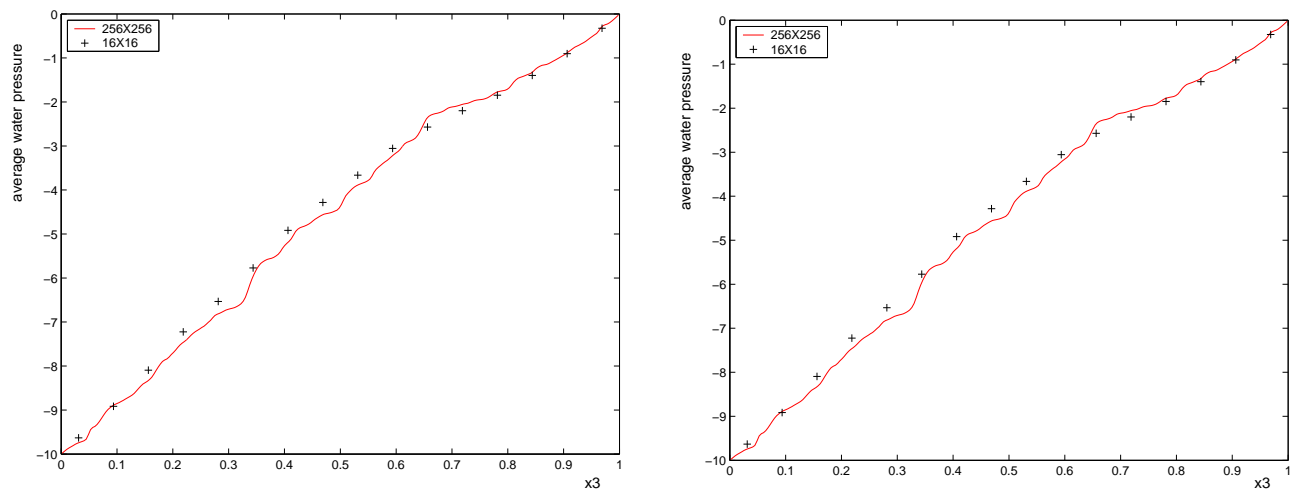

FIG. 5.14. The average solution over the horizontal direction at time $t=6$ (left) and $t=10$ (right). The solid line stands for the averaged $u_{h}^{\varepsilon}$ in a mesh $256 \times 256$, the + line stands for the averaged $\tilde{u}_{h}$ in a mesh $16 \times 16$. Case $l_{1}=0.1, l_{3}=0.01, \sigma=1.5$.

Acknowledgments. The authors would also like to thank the referees of the paper for their constructive comments which improved the paper.

\section{REFERENCES}

[1] J. Bear and Y. Bachmat, Introduction to Modelling of Transport Phenomena in Porous Media, Kluwer Academic, Dordrecht, 1991.

[2] A. Bensoussan, J. L. Lions and G. Papanicolaou, Asymptotic Analysis for Periodic Structure, Studies in Mathematics and Its Application, North-Holland Publ., 5, 1978.

[3] R. Brooks and A. Corey, Hydralic properties of porous media, Hydrology, Civil Engineering Department, Colorado State University, Fort Collins. CO, 3, 1964.

[4] Z. Chen, W. B. Deng and H. Ye, New upscaling method for the solute transport equations, Discrete and Continuous Dynamical Systems-Series A, 13, 4, 941-960, 2005.

[5] Z. Chen and T. Y. Hou, A mixed multisclae finite method for elliptic problemswith oscillating coefficients, Math. Comp., 72, 541-576, 2002.

[6] G. Christakos, Random Field Models in Earth Sciences, Academic Press, San Diego, 1992.

[7] $\mathrm{Ph}$. Clément, Approximation by finite element functions using local regularization, RAIRO 
Anal. Numer., 9, 77-84, 1975.

[8] L. J. Durlofsky, Numerical calculation of equivalent grid block permeability tensors for heterogeneous porous media, Water Resources Research, 27, 699-708, 1991.

[9] B. B. Dykaar and P. K. Kitanidis, Transmissivity of a heterogeneous formation, Water Resource Research, 29, 985-1001, 1993.

[10] W. E, P. Ming and P. Zhang, Analysis of the heterogeneous multiscale method for elliptic homogenization problems, J. AMS, to appear.

[11] Y. Efendiev, T. Y. Hou and V. Ginting, Multiscale finite element methods for nonlinear problems and their applications, Comm. Math. Sci., 2, 4, 2004.

[12] Y. Efendiev, T. Y. Hou and X. H. Wu, Convergence of a nonconforming multiscale finite element method, SIAM J. Numer. Anal., 37, 888-910, 2000.

[13] Y. Efendiev and A. Pankov, Numerical homogenization of nonlinear random parabolic operators, SIAM Multiscale Modeling and Simulation, 2, 2, 237-268, 2004.

[14] G. A. Francfort, Homogenization and linear thermoelasticity, SIAM J. Math. Anal., 14, 696$708,1983$.

[15] D. G. Fredlund and A. Xing, Equations for the soil-water characteristic curve, Canadian Geotechnical Journal, 31, 3, 521-532, 1994.

[16] N. Fusco and G. Moscariello, On the homogenization of quasilinear divergence structure operators, Ann. Mat. Pura Appl., 146, 1-13, 1987.

[17] W. R. Gardner, Some steady state solutions of unsaturared moisture flow equations with application to evaporation from a water table, Soil Sci., 85, 228-232, 1958.

[18] M. Th. van Genuchten, A closed-form equation for predicting the hydraulic conductivity of unsaturated soils, Soil. Sci. Soc. Am. J., 44, 892-898, 1980.

[19] R. Haverkamp, M. Vauclin, J. Touma, P. J. Wierenga and G.Vachaud, A comparison of numerical simulation models for one-dimensional infiltration, Soil Sci. Soc. Am. J., 41, 285-294, 1977.

[20] T. Y. Hou, X. H. Wu and Z. Cai, Convergence of a multiscale finite element method for elliptic problems with rapidly oscillation coefficients, Math. Comp., 68, 913-943, 1999.

[21] H. Jian, On the homogenization of degenerate parabolic equations, Acta Math. Appl. Sinica, 16, 100-110, 2000.

[22] V. V. Jikov, S. M. Kozlov and O. A. Oleinik, Honogenization of Differential and Integral Functionals, Springer, Berlin, 1994.

[23] O. A. Ladyzenskaja and N. N. Ural'ceva, Linear and Quasi-linear Equations Elliptic Equations, Academic Press, NewYork, 1968.

[24] A. Mantoglou and L. W. Gelhar, Stochastic modeling of large-scale transient unsaturated flow system, Water Resour. Res., 23, 1, 37-46, 1987.

[25] Y. Mualem, A new model for predicting the hydraulic conductivity of unsaturated porous media, Water Resour. Res., 12, 513-522, 1976.

[26] A. K. Nandakumaran and M. Rajesh, Homogenization of a nonlinear degenerate parabolic differential equation, Electronic Journal of Differential Equations, 17, 1-19, 2001.

[27] Ph. Renard and G. de Marsily, Calculating effective permeability: a review, Advances in Water Resources, 20, 253-278, 1997.

[28] D. Russo and M. Bouton, Statistical analysis of spatial variability in unsaturated parameters, Water Resour. Res., 28, 7, 1911-1925, 1992.

[29] M. Slodicka, A robust and efficient linearization scheme for doubly nonlinear and degenerate parabolic problems arising in flow in porous medium, SIAM J. Sci. Comput., 23, 5, 15931614, 2002.

[30] V. Thomee, Galerkin Finite Element Methods for Parabolic Problems, Springer-Verlag, Berlin, 1997.

[31] A. W. Warrick, Time-dependent linearized infiltration: III. strip and disc sources, Soil. Sci. Soc. Am. J., 40, 639-643, 1976.

[32] X. H. Wen and J. J. Gomez-Hernandez, Upscaling hydraulic conductivities in heterogeneous media, Journal of Hydrology, 183, 9-32, 1996.

[33] I. White and M. J. Sully, On the variability and use of the hydraulic conduvtivity alpha parameter in stochastic treatments of unsaturated flow, Water Resour. Res., 28, 1, 209-213, 1992.

[34] X. H. Wu, Y. Edendiev and T. Y. Hou, Analysis of upscaling absolute permeability, Discrete and Continuous Dynamical Systems-series B, 2, 185-204, 2002.

[35] T-C. J. Yeh, L. W. Gelhar and A. L. Gutjahr, Stochastic analysis of unsaturated flow in heterogeneous soils. 1. statistically isotropic media, Water Resour. Res., 21, 4, 447-456, 1985. 